\title{
Some isomorphic properties of $m$-polar fuzzy graphs with applications
}

\author{
Ganesh Ghorai* and Madhumangal Pal
}

*Correspondence: math. ganesh@mail.vidyasagar.ac.in Department of Applied Mathematics with Oceanology and Computer Programming, Vidyasagar University, Midnapore 721 102, India

\begin{abstract}
The theory of graphs are very useful tool in solving the combinatorial problems in different areas of computer science and computational intelligence systems. In this paper, we present a frame work to handle $m$-polar fuzzy information by combining the theory of $m$-polar fuzzy sets with graphs. We introduce the notion of weak self complement $m$-polar fuzzy graphs and establish a necessary condition for $m$-polar fuzzy graph to be weak self complement. Some properties of self complement and weak self complement $m$-polar fuzzy graphs are discussed. The order, size, busy vertices and free vertices of an $m$-polar fuzzy graphs are also defined and proved that isomorphic $m$-polar fuzzy graphs have same order, size and degree. Also, we have presented some results of busy vertices in isomorphic and weak isomorphic $m$-polar fuzzy graphs. Finally, a relative study of complement and operations on $m$-polar fuzzy graphs have been made. Applications of $m$-polar fuzzy graph are also given at the end.
\end{abstract}

Keywords: $m$-Polar fuzzy graphs, Order and size, Busy and free vertices, Isomorphisms, Self complement and weak self complement, 5-Polar fuzzy evaluation graph

\section{Background}

After the introduction of fuzzy sets by Zadeh (1965), fuzzy set theory have been included in many research fields. Since then, the theory of fuzzy sets has become a vigorous area of research in different disciplines including medical and life sciences, management sciences, social sciences engineering, statistic, graph theory, artificial intelligence, signal processing, multi agent systems, decision making and automata theory. In a fuzzy set, each element is associated with a membership value selected from the interval $[0,1]$. Zhang $(1994,1998)$ introduced the concept of bipolar fuzzy sets. Instead of using particular membership value as in fuzzy sets, $m$-polar fuzzy set can be used to represent uncertainty of a set more perfectly. Chen et al. (2014) introduced the notion of $m$-polar fuzzy set as a generalization of fuzzy set theory. The membership value in $m$-polar fuzzy set is more expressive in capturing uncertainty of data.

An $m$-polar fuzzy set on a non-void set $X$ is a mapping $\mu: X \rightarrow[0,1]^{m}$. The idea behind this is that "multipolar information" exists because data of real world problems are sometimes come from multiple agents. $m$-polar fuzzy sets allow more graphical representation of vague data, which facilitates significantly better analysis in data relationships, incompleteness, and similarity measures. Graph theory besides being a well developed branch of Mathematics, it is an important tool for mathematical modeling.

(c) The Author(s) 2016. This article is distributed under the terms of the Creative Commons Attribution 4.0 International License (http://creativecommons.org/licenses/by/4.0/), which permits unrestricted use, distribution, and reproduction in any medium, provided you give appropriate credit to the original author(s) and the source, provide a link to the Creative Commons license, and indicate if changes were made. 
Realizing the importance, Rosenfeld (1975) introduced the concept of fuzzy graphs, Mordeson and Nair (2000) discussed about the properties of fuzzy graphs and hypergraphs. After that, the operation of union, join, Cartesian product and composition on two fuzzy graphs was defined by Mordeson and Peng (1994). Sunitha and Vijayakumar (2002) further studied the other properties of fuzzy graphs. The concept of weak isomorphism, co-weak isomorphism and isomorphism between fuzzy graphs was introduced by Bhutani (1989). Later many researchers have worked on fuzzy graphs like in Bhutani et al. (2004); Al-Hawary (2011); Koczy (1992); Lee-kwang and Lee (1995); Nagoorgani and Radha (2008), Samanta and Pal (2011a, b, 2013, 2014, 2015). Akram (2011, 2013) introduced and defined different operations on bipolar fuzzy graphs. Again, Rashmanlou et al. (2015a, 2015b, 2016) studied bipolar fuzzy graphs with categorical properties, product of bipolar fuzzy graphs and their degrees, etc. Using these concepts many research is going on till date on bipolar fuzzy graphs such as Ghorai and Pal (2015b), Samanta and Pal (2012a, b, 2014), Yang et al. (2013). Chen et al. (2014) first introduced the concept of $m$-polar fuzzy graphs. Then Ghorai and Pal (2016a) presented properties of generalized $m$-polar fuzzy graphs, defined many operations and density of $m$-polar fuzzy graphs (2015a), introduced the concept of $m$-polar fuzzy planar graphs (2016b) and defined faces and dual of $m$-polar fuzzy planar graphs (2016c). Akram and Younas (2015), Akram et al. (2016) introduced irregular $m$-polar fuzzy graphs and metrics in $m$-polar fuzzy graphs. In this paper, weak self complement $m$-polar fuzzy graphs is defined and a necessary condition is mentioned for an $m$-polar fuzzy graph to be weak self complement. Some properties of self complement and weak self complement $m$-polar fuzzy graphs are discussed. The order, size, busy vertices and free vertices of an $m$-polar fuzzy graphs are also defined and proved that isomorphic $m$-polar fuzzy graphs have same order, size and degree. Also, we have proved some results of busy vertices in isomorphic and weak isomorphic $m$-polar fuzzy graphs. Finally, a relative study of complement and operations on $m$-polar fuzzy graphs have been made.

\section{Preliminaries}

First of all we give the definitions of $m$-polar fuzzy sets, $m$-polar fuzzy graphs and other related definitions from the references (Al-Harary 1972; Lee 2000).

Throughout the paper, $[0,1]^{m}$ (m-power of $\left.[0,1]\right)$ is considered to be a poset with point-wise order $\leq$ where $m$ is a natural number. $\leq$ is defined by $x \leq y \Leftrightarrow$ for each $i=1,2, \ldots, m, p_{i}(x) \leq p_{i}(y)$ where $x, y \in[0,1]^{m}$ and $p_{i}:[0,1]^{m} \rightarrow[0,1]$ is the $i$ th projection mapping.

As a generalization of bipolar fuzzy sets, Chen et al. (2014) defined the $m$-polar fuzzy sets in 2014.

Definition 1 (Chen et al. 2014) Let $X$ be a non-void set. An $m$-polar fuzzy set on $X$ is defined as a mapping $\mu: X \rightarrow[0,1]^{m}$.

The $m$-polar fuzzy relation is defined below.

Definition 2 (Ghorai and Pal 2016a) Let $A$ be an $m$-polar fuzzy set on a set $X$. An $m$-polar fuzzy relation on $A$ is an $m$-polar fuzzy set $B$ of $X \times X$ such that 
$p_{i} \circ B(x, y) \leq \min \left\{p_{i} \circ A(x), p_{i} \circ A(y)\right\}$ for all $x, y \in X, i=1,2, \ldots, m . B$ is called symmetric if $B(x, y)=B(y, x)$ for all $x, y \in X$.

We define an equivalence relation $\sim$ on $V \times V-\{(x, x): x \in V\}$ as follows:

We say $\left(x_{1}, y_{1}\right) \sim\left(x_{2}, y_{2}\right)$ if and only if either $\left(x_{1}, y_{1}\right)=\left(x_{2}, y_{2}\right)$ or $x_{1}=y_{2}$ and $y_{1}=x_{2}$. Then we obtain an quotient set denoted by $\widetilde{V^{2}}$. The equivalence class containing the element $(x, y)$ will be denoted as $x y$ or $y x$.

We assume that $G^{*}=(V, E)$ is a crisp graph and $G=(V, A, B)$ is an $m$-polar fuzzy graph of $G^{*}$ throughout this paper.

Chen et al. (2014) first introduced $m$-polar fuzzy graph. We have modified their definition and introduce generalized $m$-polar fuzzy graph as follows.

Definition 3 (Chen et al. 2014; Ghorai and Pal 2016a) An $m$-polar fuzzy graph (or generalized $m$-polar fuzzy graph) of $G^{*}=(V, E)$ is a pair $G=(V, A, B)$ where $A: V \rightarrow[0,1]^{m}$ is an $m$-polar fuzzy set in $V$ and $B: \widetilde{V^{2}} \rightarrow[0,1]^{m}$ is an $m$-polar fuzzy set in $\widetilde{V^{2}}$ such that $p_{i} \circ B(x y) \leq \min \left\{p_{i} \circ A(x), p_{i} \circ A(y)\right\}$ for all $x y \in \widetilde{V^{2}}, i=1,2, \ldots, m$ and $B(x y)=\mathbf{0}$ for all $x y \in \widetilde{V^{2}}-E,\left(\mathbf{0}=(0,0, \ldots, 0)\right.$ is the smallest element in $\left.[0,1]^{m}\right)$. We call $A$ as the $m$-polar fuzzy vertex set of $G$ and $B$ as the $m$-polar fuzzy edge set of $G$.

Example 4 Let $G^{*}=(V, E)$ be a crisp graph where $V=\left\{u_{1}, u_{2}, u_{3}, u_{4}\right\}$ and $E=\left\{u_{1} u_{2}, u_{2} u_{3}, u_{3} u_{4}, u_{4} u_{1}\right\}$. Then, $G=(V, A, B)$ be a 3-polar fuzzy graph of $G^{*}$ where $A=\left\{\frac{\langle 0.5,0.7,0.8\rangle}{u_{1}}, \frac{\langle 0.4,0.7,0.8\rangle}{u_{2}}, \frac{\langle 0.7,0.6,0.8\rangle}{u_{3}}, \frac{\langle 0.3,0.6,0.9\rangle}{u_{4}}\right\}$ and $B=\left\{\frac{\langle 0.4,0.6,0.7\rangle}{u_{1} u_{2}}, \frac{\langle 0.3,0.6,0.5\rangle}{u_{2} u_{3}}\right.$, $\left.\frac{\langle 0.2,0.5,0.6\rangle}{u_{3} u_{4}}, \frac{\langle 0.2,0.4,0.8\rangle}{u_{4} u_{1}}, \frac{\langle 0,0,0\rangle}{u_{1} u_{3}}, \frac{\langle 0,0,0\rangle}{u_{4} u_{2}}\right\}$.

Ghorai and Pal (2016a) introduced many operations on $m$-polar fuzzy graphs such as Cartesian product, composition, union and join which are given below.

Definition 5 (Ghorai and Pal 2016a) The Cartesian product of two $m$-polar fuzzy graphs $G_{1}=\left(V_{1}, A_{1}, B_{1}\right)$ and $G_{2}=\left(V_{2}, A_{2}, B_{2}\right)$ of the graphs $G_{1}^{*}$ and $G_{2}^{*}$ respectively is denoted as a pair $G_{1} \times G_{2}=\left(V_{1} \times V_{2}, A_{1} \times A_{2}, B_{1} \times B_{2}\right)$ such that for $i=1,2, \ldots, m$

(i) $\quad p_{i} \circ\left(A_{1} \times A_{2}\right)\left(x_{1}, x_{2}\right)=\min \left\{p_{i} \circ A_{1}\left(x_{1}\right), p_{i} \circ A_{2}\left(x_{2}\right)\right\}$ for all $\left(x_{1}, x_{2}\right) \in V_{1} \times V_{2}$.

(ii) $p_{i} \circ\left(B_{1} \times B_{2}\right)\left(\left(x, x_{2}\right)\left(x, y_{2}\right)\right)=\min \left\{p_{i} \circ A_{1}(x), p_{i} \circ B_{2}\left(x_{2} y_{2}\right)\right\}$ for all $x \in V_{1}$, $x_{2} y_{2} \in E_{2}$.

(iii) $p_{i} \circ\left(B_{1} \times B_{2}\right)\left(\left(x_{1}, z\right)\left(y_{1}, z\right)\right)=\min \left\{p_{i} \circ B_{1}\left(x_{1} y_{1}\right), p_{i} \circ A_{2}(z)\right\}$ for all $z \in V_{2}$, $x_{1} y_{1} \in E_{1}$.

(iv) $p_{i} \circ\left(B_{1} \times B_{2}\right)\left(\left(x_{1}, x_{2}\right)\left(y_{1}, y_{2}\right)\right)=0$ for all $\left(x_{1}, x_{2}\right)\left(y_{1}, y_{2}\right) \in\left(\widetilde{V_{1} \times V_{2}}\right)^{2}-E$.

Definition 6 (Ghorai and Pal 2016a) The composition of two $m$-polar fuzzy graphs $G_{1}=\left(V_{1}, A_{1}, B_{1}\right)$ and $G_{2}=\left(V_{2}, A_{2}, B_{2}\right)$ of the graphs $G_{1}^{*}=\left(V_{1}, E_{1}\right)$ and $G_{2}^{*}=\left(V_{2}, E_{2}\right)$ respectively is denoted as a pair $G_{1}\left[G_{2}\right]=\left(V_{1} \times V_{2}, A_{1} \circ A_{2}, B_{1} \circ B_{2}\right)$ such that for $i=1,2, \ldots, m$ 
(i) $p_{i} \circ\left(A_{1} \circ A_{2}\right)\left(x_{1}, x_{2}\right)=\min \left\{p_{i} \circ A_{1}\left(x_{1}\right), p_{i} \circ A_{2}\left(x_{2}\right)\right\}$ for all $\left(x_{1}, x_{2}\right) \in V_{1} \times V_{2}$.

(ii) $p_{i} \circ\left(B_{1} \circ B_{2}\right)\left(\left(x, x_{2}\right)\left(x, y_{2}\right)\right)=\min \left\{p_{i} \circ A_{1}(x), p_{i} \circ B_{2}\left(x_{2} y_{2}\right)\right\}$ for all $x \in V_{1}$, $x_{2} y_{2} \in E_{2}$.

(iii) $p_{i} \circ\left(B_{1} \circ B_{2}\right)\left(\left(x_{1}, z\right)\left(y_{1}, z\right)\right)=\min \left\{p_{i} \circ B_{1}\left(x_{1} y_{1}\right), p_{i} \circ A_{2}(z)\right\} \quad$ for all $z \in V_{2}$, $x_{1} y_{1} \in E_{1}$.

(iv) $p_{i} \circ\left(B_{1} \circ B_{2}\right)\left(\left(x_{1}, x_{2}\right)\left(y_{1}, y_{2}\right)\right)=\min \left\{p_{i} \circ A_{2}\left(x_{2}\right), p_{i} \circ A_{2}\left(y_{2}\right), p_{i} \circ B_{1}\left(x_{1} y_{1}\right)\right\}$ for all $\left(x_{1}, x_{2}\right)\left(y_{1}, y_{2}\right) \in E^{0}-E$.

(v) $p_{i} \circ\left(B_{1} \circ B_{2}\right)\left(\left(x_{1}, x_{2}\right)\left(y_{1}, y_{2}\right)\right)=0$ for all $\left(x_{1}, x_{2}\right)\left(y_{1}, y_{2}\right) \in\left(\widetilde{V_{1} \times V_{2}}\right)^{2}-E^{0}$.

Definition 7 (Ghorai and Pal 2016a) The union $G_{1} \cup G_{2}=\left(V_{1} \cup V_{2}, A_{1} \cup A_{2}, B_{1} \cup B_{2}\right)$ of the $m$-polar fuzzy graphs $G_{1}=\left(V_{1}, A_{1}, B_{1}\right)$ and $G_{2}=\left(V_{2}, A_{2}, B_{2}\right)$ of $G_{1}^{*}$ and $G_{2}^{*}$ respectively is defined as follows: for $i=1,2, \ldots, m$
(i) $p_{i} \circ\left(A_{1} \cup A_{2}\right)(x)=\left\{\begin{array}{l}p_{i} \circ A_{1}(x) \\ p_{i} \circ A_{2}(x) \\ \max \left\{p_{i} \circ A_{1}(x), p_{i} \circ A_{2}(x)\right\}\end{array}\right.$
if $x \in V_{1}-V_{2}$
if $x \in V_{2}-V_{1}$
if $x \in V_{1} \cap V_{2}$.
(ii) $p_{i} \circ\left(B_{1} \cup B_{2}\right)(x y)=\left\{\begin{array}{l}p_{i} \circ B_{1}(x y) \\ p_{i} \circ B_{2}(x y) \\ \max \left\{p_{i} \circ B_{1}(x y), p_{i} \circ B_{2}(x y)\right\}\end{array}\right.$
if $x y \in E_{1}-E_{2}$
if $x y \in E_{2}-E_{1}$
if $x y \in E_{1} \cap E_{2}$.
(iii) $p_{i} \circ\left(B_{1} \cup B_{2}\right)(x y)=0$ if $x y \in\left(\widetilde{V_{1} \times V_{2}}\right)^{2}-E_{1} \cup E_{2}$.

Definition 8 (Ghorai and Pal 2016a) The join of the $m$-polar fuzzy graphs $G_{1}=\left(V_{1}, A_{1}, B_{1}\right)$ and $G_{2}=\left(V_{2}, A_{2}, B_{2}\right)$ of $G_{1}^{*}$ and $G_{2}^{*}$ respectively is defined as a pair $G_{1}+G_{2}=\left(V_{1} \cup V_{2}, A_{1}+A_{2}, B_{1}+B_{2}\right)$ such that for $i=1,2, \ldots, m$

(i) $p_{i} \circ\left(A_{1}+A_{2}\right)(x)=p_{i} \circ\left(A_{1} \cup A_{2}\right)(x)$ if $x \in V_{1} \cup V_{2}$.

(ii) $p_{i} \circ\left(B_{1}+B_{2}\right)(x y)=p_{i} \circ\left(B_{1} \cup B_{2}\right)(x y)$ if $x y \in E_{1} \cup E_{2}$.

(iii) $p_{i} \circ\left(B_{1}+B_{2}\right)(x y)=\min \left\{p_{i} \circ A_{1}(x), p_{i} \circ A_{2}(y)\right\}$ if $x y \in E^{\prime}$, where $E^{\prime}$ denotes the set of all edges joining the vertices of $V_{1}$ and $V_{2}$.

(iv) $p_{i} \circ\left(B_{1}+B_{2}\right)(x y)=0$ if $x y \in\left(V_{1} \times V_{2}\right)^{2}-E_{1} \cup E_{2} \cup E^{\prime}$.

Remark 9 Later on, Akram et al. (2016) applied the concept of $m$-polar fuzzy sets on graph structure and also defined the above operations on them.

Different types of morphism are defined on $m$-polar fuzzy graphs by Ghorai and Pal (2016a).

Definition 10 (Ghorai and Pal 2016a) Let $G_{1}=\left(V_{1}, A_{1}, B_{1}\right)$ and $G_{2}=\left(V_{2}, A_{2}, B_{2}\right)$ be two $m$-polar fuzzy graphs of the graphs $G_{1}^{*}=\left(V_{1}, E_{1}\right)$ and $G_{2}^{*}=\left(V_{2}, E_{2}\right)$ respectively. A homomorphism between $G_{1}$ and $G_{2}$ is a mapping $\phi: V_{1} \rightarrow V_{2}$ such that for each $i=1,2, \ldots, m$

(i) $p_{i} \circ A_{1}\left(x_{1}\right) \leq p_{i} \circ A_{2}\left(\phi\left(x_{1}\right)\right)$ for all $x_{1} \in V_{1}$,

(ii) $p_{i} \circ B_{1}\left(x_{1} y_{1}\right) \leq p_{i} \circ B_{2}\left(\phi\left(x_{1}\right) \phi\left(y_{1}\right)\right)$ for all $x_{1} y_{1} \in \widetilde{V_{1}^{2}}$. 
$\phi$ is said to be an isomorphism if it is a bijective mapping and for $i=1,2, \ldots, m$

(i) $p_{i} \circ A_{1}\left(x_{1}\right)=p_{i} \circ A_{2}\left(\phi\left(x_{1}\right)\right)$ for all $x_{1} \in V_{1}$,

(ii) $p_{i} \circ B_{1}\left(x_{1} y_{1}\right)=p_{i} \circ B_{2}\left(\phi\left(x_{1}\right) \phi\left(y_{1}\right)\right)$ for all $x_{1} y_{1} \in \widetilde{V_{1}^{2}}$.

In this case, we write $G_{1} \cong G_{2}$.

Definition 11 (Ghorai and Pal 2016a) A weak isomorphism between $G_{1}=\left(V_{1}, A_{1}, B_{1}\right)$ and $G_{2}=\left(V_{2}, A_{2}, B_{2}\right)$ is a bijective mapping $\phi: V_{1} \rightarrow V_{2}$ such that

(i) $\phi$ is a homomorphism,

(ii) $p_{i} \circ A_{1}\left(x_{1}\right)=p_{i} \circ A_{2}\left(\phi\left(x_{1}\right)\right)$ for all $x_{1} \in V_{1}$, for each $i=1,2, \ldots, m$.

Definition 12 (Ghorai and Pal 2016a) $G=(V, A, B)$ is called strong if $p_{i} \circ B(x y)=\min \left\{p_{i} \circ A(x), p_{i} \circ A(y)\right\}$ for all $x y \in E, i=1,2, \ldots, m$.

A strong $m$-polar fuzzy graph $G$ is called self complementary if $G \cong \bar{G}$.

Degree of a vertex in an $m$-polar fuzzy graph is defined as below.

Definition 13 (Akram and Younas 2015) The neighborhood degree of a vertex $v$ in the $m$-polar fuzzy graph $G$ is denoted as $\operatorname{deg}(v)=\left(p_{1} \circ \operatorname{deg}(v), p_{2} \circ \operatorname{deg}(v), \ldots, p_{m} \circ \operatorname{deg}(v)\right)$ where $p_{i} \circ \operatorname{deg}(v)=\sum_{u \neq v} p_{i} \circ B(u v), i=1,2, \ldots, m$. $u v \in E$

Remark 14 If $G_{1}=\left(V_{1}, A_{1}, B_{1}\right)$ and $G_{2}=\left(V_{2}, A_{2}, B_{2}\right)$ are two $m$-polar fuzzy graphs. Then the canonical projection maps $\pi_{1}: V_{1} \times V_{2} \rightarrow V_{1}$ and $\pi_{2}: V_{1} \times V_{2} \rightarrow V_{2}$ are indeed homomorphisms from $G_{1} \times G_{2}$ to $G_{1}$ and $G_{1} \times G_{2}$ to $G_{2}$ respectively. This can be seen as follows:

$$
p_{i} \circ\left(A_{1} \times A_{2}\right)\left(x_{1}, x_{2}\right)=\min \left\{p_{i} \circ A_{1}\left(x_{1}\right), p_{i} \circ A_{2}\left(x_{2}\right)\right\} \leq p_{i} \circ A_{1}\left(x_{1}\right)=p_{i} \circ A_{1}\left(\pi_{1}\left(x_{1}, x_{2}\right)\right) \quad \text { for }
$$
all $\left(x_{1}, x_{2}\right) \in V_{1} \times V_{2}$ and $p_{i} \circ\left(B_{1} \times B_{2}\right)\left(\left(x_{1}, z\right)\left(y_{1}, z\right)\right)=\min \left\{p_{i} \circ B_{1}\left(x_{1} y_{1}\right), p_{i} \circ A_{2}(z)\right\} \leq$ $p_{i} \circ B_{1}\left(x_{1} y_{1}\right)=p_{i} \circ B_{1}\left(\pi_{1}\left(x_{1}, z\right) \pi_{1}\left(y_{1}, z\right)\right)$ for all $z \in V_{2}$ and $x_{1} y_{1} \in E_{1}$. In a similar way we can check the other conditions also. This shows that the canonical projection maps $\pi_{1}: V_{1} \times V_{2} \rightarrow V_{1}$ is a homomorphism from $G_{1} \times G_{2}$ to $G_{1}$.

\section{Weak self complement $\boldsymbol{m}$-polar fuzzy graphs}

Self complement $m$-polar fuzzy graphs have many important significant in the theory of $m$-polar fuzzy graphs. If an $m$-polar fuzzy graph is not self complement then also we can say that it is self complement in some weaker sense. Simultaneously we can establish some results with this graph. This motivates to define weak self complement $m$-polar fuzzy graphs.

Definition 15 Let $G=(V, A, B)$ be an $m$-polar fuzzy graph of the crisp graph $G^{*}=(V, E)$. The complement of $G$ is an $m$-polar fuzzy graph $\bar{G}=(V, \bar{A}, \bar{B})$ of $\overline{G^{*}}=\left(V, \widetilde{V^{2}}\right)$ such that $\bar{A}=A$ and $\bar{B}$ is defined by $p_{i} \circ \bar{B}(x y)=\min \left\{p_{i} \circ A(x), p_{i} \circ A(y)\right\}-p_{i} \circ B(x y)$ for $x y \in \widetilde{V^{2}}, i=1,2, \ldots, m$. 
Example 16 Let $G=(V, A, B)$ be a 3-polar fuzzy graph of the graph $G^{*}=(V, E)$ where $V=\{u, v, w, x\}, E=\{u v, v w, w u, u x\}, A=\left\{\frac{\langle 0.2,0.3,0.5\rangle}{u}, \frac{\langle 0.5,0.6,0.3\rangle}{v}, \frac{\langle 0.7,0.2,0.3\rangle}{w}, \frac{\langle 0.2,0.5,0.7\rangle}{x}\right\}$, $B=\left\{\frac{\langle 0.2,0.3,0.3\rangle}{u v}, \frac{\langle 0.4,0.1,0.1\rangle}{v w}, \frac{\langle 0.1,0.1,0.1\rangle}{w u}, \frac{\langle 0.1,0.2,0.4\rangle}{x u}, \frac{\langle 0,0,0\rangle}{x v}, \frac{\langle 0,0,0\rangle}{w x}\right\}$. Then by Definition 15, we have constructed the complement $\bar{G}$ of $G$ which is shown in Fig. 1.

Remark 17 Let $\overline{\bar{G}}=(V, \overline{\bar{A}}, \overline{\bar{B}})$ be the complement of $\bar{G}$ where $\overline{\bar{A}}=\bar{A}=A$ and

$$
\begin{aligned}
p_{i} \circ \overline{\bar{B}}(u v) & =\min \left\{p_{i} \circ \bar{A}(u), p_{i} \circ \bar{A}(v)\right\}-p_{i} \circ \bar{B}(u v) \\
& =\min \left\{p_{i} \circ A(u), p_{i} \circ A(v)\right\}-\left\{\min \left\{p_{i} \circ A(u), p_{i} \circ A(v)\right\}-p_{i} \circ B(u v)\right\} \\
& =p_{i} \circ B(u v) \quad \text { for } u v \in \widetilde{V^{2}}, i=1,2, \ldots, m .
\end{aligned}
$$

Hence, $\overline{\bar{G}}=G$.

Definition 18 The $m$-polar fuzzy graph $G=(V, A, B)$ is said to be weak self complement if there is a weak isomorphism from $G$ onto $\bar{G}$. In other words, there exist a bijective homomorphism $\phi: G \rightarrow \bar{G}$ such that for $i=1,2, \ldots, m$

(i) $\quad p_{i} \circ A(u)=p_{i} \circ \bar{A}(\phi(u))$ for all $u \in V$,

(ii) $\quad p_{i} \circ B(u v) \leq p_{i} \circ \bar{B}(\phi(u) \phi(v))$ for all $u v \in \widetilde{V^{2}}$.

Example 19 Let $G=(V, A, B)$ be a 3-polar fuzzy graph of the graph $G^{*}=(V, E)$ where $\quad V=\{u, v, w\}, \quad E=\{u v, v w\}, \quad A=\left\{\frac{\langle 0.3,0.4,0.4\rangle}{u}, \frac{\langle 0.2,0.5,0.7\rangle}{v}, \frac{\langle 0.3,0.6,0.7\rangle}{w}\right\}$, $B=\left\{\frac{\langle 0.1,0.1,0.2\rangle}{u v}, \frac{\langle 0.1,0.2,0.2\rangle}{v w}, \frac{\langle 0,0,0\rangle}{w u}\right\}$. Then $\bar{G}=(V, \bar{A}, \bar{B})$ is also a 3-polar fuzzy graph where $\bar{A}=A$ and $\bar{B}=\left\{\frac{\langle 0.1,0.3,0.2\rangle}{u v}, \frac{\langle 0.1,0.3,0.5\rangle}{v w}, \frac{\langle 0.3,0.4,0.4\rangle}{w u}\right\}$. We can easily verify that, the identity map is an weak isomorphism from $G$ onto $\bar{G}$ (see Fig. 2). Hence $G$ is weak self complement.

In Ghorai and Pal (2015a), Ghorai and Pal proved that if $G$ is a self complementary strong $m$-polar fuzzy graph then for all $x y \in \widetilde{V^{2}}$ and $i=1,2, \ldots, m$

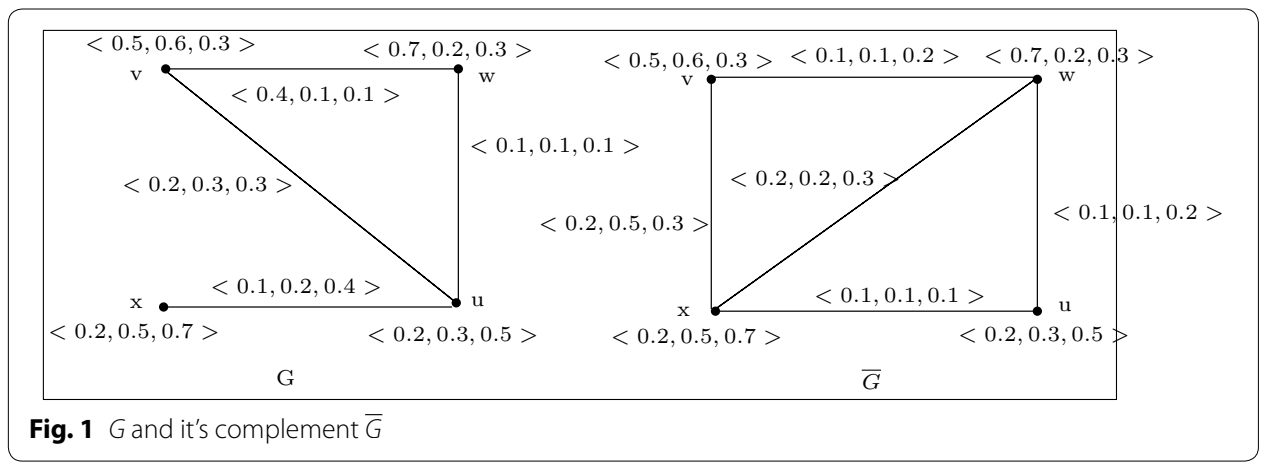




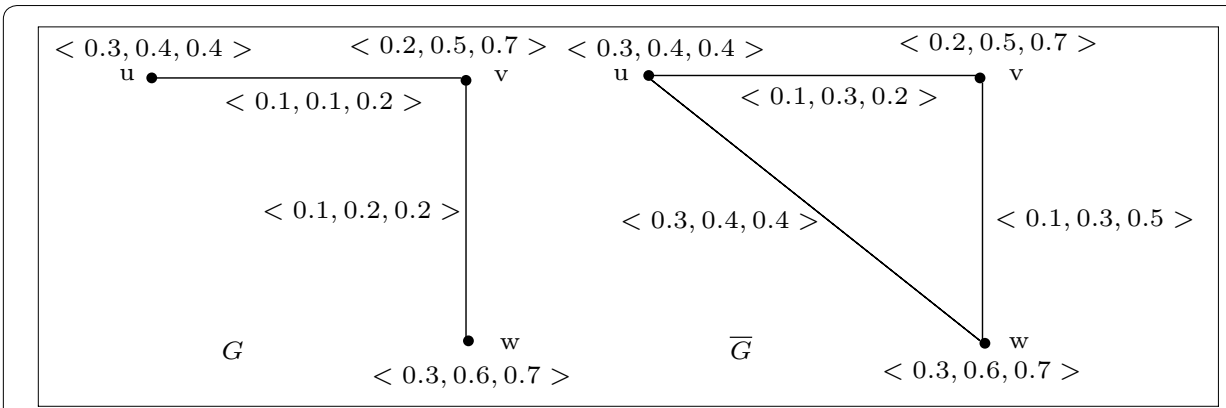

Fig. 2 Weak self complement 3-polar fuzzy graphs

$$
\sum_{x \neq y} p_{i} \circ B(x y)=\frac{1}{2} \sum_{x \neq y} \min \left\{p_{i} \circ A(x), p_{i} \circ A(y)\right\} .
$$

The converse of the above result does not hold always.

Example 20 For example, let us consider a 3-polar fuzzy graph $G=(V, A, B)$ of $G^{*}=(V, E)$ where $V=\{u, v, w\}, E=\{u v, v w, w u\}, A=\left\{\frac{\langle 0.2,0.3,0.4\rangle}{u}, \frac{\langle 0.4,0.5,0.6\rangle}{v}, \frac{\langle 0.5,0.7,0.8\rangle}{w}\right\}$, $B=\left\{\frac{\langle 0.2,0.3,0.4\rangle}{u v}, \frac{\langle 0.1,0.2,0.2\rangle}{v w}, \frac{\langle 0.1,0.05,0.1\rangle}{w u}\right\}$. Then we have the following

$$
\begin{aligned}
& p_{1} \circ B(u v)+p_{1} \circ B(v w)+p_{1} \circ B(w u)=0.2+0.1+0.1=0.4 \text { and } \\
& \frac{1}{2}\left[\min \left\{p_{1} \circ A(u), p_{1} \circ A(v)\right\}+\min \left\{p_{1} \circ A(v), p_{1} \circ A(w)\right\}+\min \left\{p_{1} \circ A(w), p_{1} \circ A(u)\right\}\right] \\
& \quad=\frac{1}{2}[\min \{0.2,0.4\}+\min \{0.4,0.5\}+\min \{0.5,0.2\}]=\frac{1}{2}(0.2+0.4+0.2)=0.4 .
\end{aligned}
$$

So,

$$
\sum_{u \neq v} p_{1} \circ B(u v)=0.4=\frac{1}{2} \sum_{u \neq v} \min \left\{p_{1} \circ A(u), p_{i} \circ A(v)\right\}
$$

Similarly,

$$
\sum_{u \neq v} p_{2} \circ B(u v)=0.55=\frac{1}{2} \sum_{u \neq v} \min \left\{p_{2} \circ A(u), p_{2} \circ A(v)\right\}
$$

and

$$
\sum_{u \neq v} p_{3} \circ B(u v)=0.7=\frac{1}{2} \sum_{u \neq v} \min \left\{p_{3} \circ A(u), p_{3} \circ A(v)\right\} .
$$


Hence for $i=1,2,3$ we have,

$$
\sum_{u \neq v} p_{i} \circ B(u v)=\frac{1}{2} \sum_{u \neq v} \min \left\{p_{i} \circ A(u), p_{i} \circ A(v)\right\} .
$$

But $G$ is not self complementary as there exists no isomorphism from $G$ onto $\bar{G}$ (see Fig. 3).

Now suppose an $m$-polar fuzzy graph $G=(V, A, B)$ is a weak self complement. Then the following inequality holds.

Theorem 21 Let $G=(V, A, B)$ be a weak self complement m-polar fuzzy graph of $G^{*}$.

Then for $i=1,2, \ldots, m$

$$
\sum_{x \neq y} p_{i} \circ B(x y) \leq \frac{1}{2} \sum_{x \neq y} \min \left\{p_{i} \circ A(x), p_{i} \circ A(y)\right\}
$$

Proof Since $G$ is weak self complement, therefore there exists a weak isomorphism $\phi: V \rightarrow V$ such that $p_{i} \circ A(x)=p_{i} \circ \bar{A}(\phi(x))$ for all $x \in V$ and $p_{i} \circ B(x y) \leq p_{i} \circ \bar{B}(\phi(x) \phi(y))$ for all $x y \in \widetilde{V^{2}}, i=1,2, \ldots, m$.

Using the above we have,

$$
\begin{aligned}
& p_{i} \circ B(x y) \leq p_{i} \circ \bar{B}(\phi(x) \phi(y))=\min \left\{p_{i} \circ A(x), p_{i} \circ A(y)\right\}-p_{i} \circ B(\phi(x) \phi(y)) \\
& \text { i.e., } p_{i} \circ B(x y)+p_{i} \circ B(\phi(x) \phi(y)) \leq \min \left\{p_{i} \circ A(\phi(x)), p_{i} \circ A(\phi(y))\right\} .
\end{aligned}
$$

Therefore, for all $x y \in \widetilde{V^{2}}, i=1,2, \ldots, m$

$$
\begin{aligned}
\sum_{x \neq y} p_{i} \circ B(x y)+\sum_{x \neq y} p_{i} \circ B(\phi(x) \phi(y) \\
\leq \sum_{x \neq y} \min \left\{p_{i} \circ A(\phi(x)), p_{i} \circ A(\phi(y))\right\} \\
=\sum_{x \neq y} \min \left\{p_{i} \circ A(x), p_{i} \circ A(y)\right\}
\end{aligned}
$$

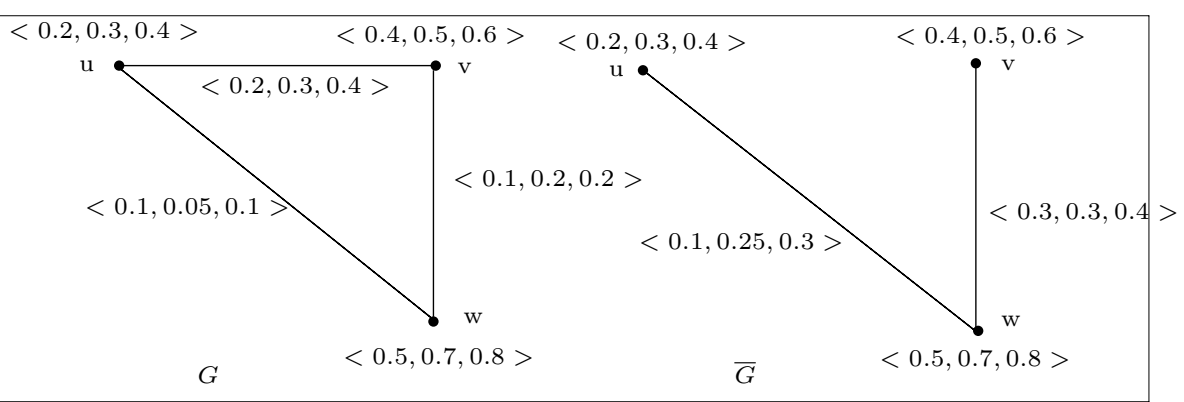

Fig. 3 Example of 3-polar fuzzy graph $G$ which is not self complement 
i.e.,

$$
2 \sum_{x \neq y} p_{i} \circ B(x y) \leq \sum_{x \neq y} \min \left\{p_{i} \circ A(x), p_{i} \circ A(y)\right\}
$$

i.e.,

$$
\sum_{x \neq y} p_{i} \circ B(x y) \leq \frac{1}{2} \sum_{x \neq y} \min \left\{p_{i} \circ A(x), p_{i} \circ A(y)\right\} .
$$

Remark 22 The converse of the above theorem is not true in general. For example, consider the 3-polar fuzzy graph of Fig. 3. We see that for the 3-polar fuzzy graph $G$, the condition of Theorem 21 is satisfied. But, $G$ is not weak self complementary as there is no weak isomorphism from $G$ onto $\bar{G}$.

Theorem 23 If $p_{i} \circ B(x y) \leq \frac{1}{2} \min \left\{p_{i} \circ A(x), p_{i} \circ A(y)\right\}$ for all $x y \in \widetilde{V^{2}}, i=1,2, \ldots, m$ then $G$ is a weak self complement m-polar fuzzy graph.

Proof Let $\bar{G}=(V, \bar{A}, \bar{B})$ be the complement of $G$ where $\bar{A}(x)=A(x)$ for all $x \in V$ and $p_{i} \circ \bar{B}(x y)=\min \left\{p_{i} \circ A(x), p_{i} \circ A(y)\right\}-p_{i} \circ B(x y)$ for $x y \in \widetilde{V^{2}}, i=1,2, \ldots, m$.

Let us now consider the identity map $I: V \rightarrow V$. Then $A(x)=A(I(x))=\bar{A}(I(x))$ for all $x \in V$ and

$$
\begin{aligned}
p_{i} \circ \bar{B}(I(x) I(y)) & =p_{i} \circ \bar{B}(x y) \\
& =\min \left\{p_{i} \circ A(x), p_{i} \circ A(y)\right\}-p_{i} \circ B(x y) \\
& \geq \min \left\{p_{i} \circ A(x), p_{i} \circ A(y)\right\}-\frac{1}{2} \min \left\{p_{i} \circ A(x), p_{i} \circ A(y)\right\} \\
& =\frac{1}{2} \min \left\{p_{i} \circ A(x), p_{i} \circ A(y)\right\} \geq p_{i} \circ B(x y) .
\end{aligned}
$$

So, $p_{i} \circ B(x y) \leq p_{i} \circ \bar{B}(I(x) I(y))$ for $i=1,2, \ldots, m$ and $x y \in \widetilde{V^{2}}$. Hence, $I: V \rightarrow V$ is a weak isomorphism.

Example 24 Consider the 3-polar fuzzy graph $G=(V, A, B)$ of $G^{*}=(V, E)$ where $V=\{u, v, w\}, \quad E=\{u v, v w, w u\}, \quad A=\left\{\frac{\langle 0.2,0.3,0.4\rangle}{u}, \frac{\langle 0.4,0.5,0.6\rangle}{v}, \frac{\langle 0.5,0.7,0.9\rangle}{w}\right\}$, $B=\left\{\frac{\langle 0.1,0.1,0.2\rangle}{u v}, \frac{\langle 0.2,0.2,0.3\rangle}{v w}, \frac{\langle 0.1,0.1,0.2\rangle}{w u}\right\}$. We see that for each $i=1,2,3$ and $x y \in \widetilde{V^{2}}$, $p_{i} \circ B(x y) \leq \frac{1}{2} \min \left\{p_{i} \circ A(x), p_{i} \circ A(y)\right\}$.

Also, consider the complement of $G$ of Fig. 4. Let us now consider the identity mapping $I: G \rightarrow \bar{G}$ such that $I(u)=u$ for all $u \in V$. Then, $I$ is the required weak isomorphism from $G$ onto $\bar{G}$. Hence, $G$ is weak self complementary.

\section{Order, size and busy value of vertices of $\boldsymbol{m}$-polar fuzzy graphs}

In this section, the order, size, busy value of vertices of an $m$-polar fuzzy graph is defined. 


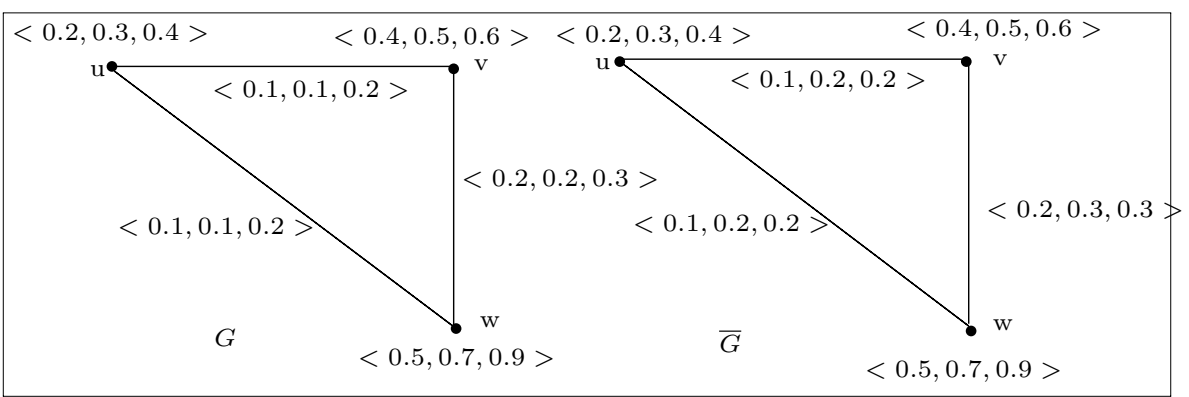

Fig. 4 Example of 3-polar fuzzy graph $G$ which is weak self complement

Definition 25 The order of the $m$-polar fuzzy graph $G=(V, A, B)$ is denoted by $|V|$ (or $O(G)$ ) where

$$
O(G)=|V|=\sum_{x \in V} \frac{1+\sum_{i=1}^{m} p_{i} \circ A(x)}{2} .
$$

The size of $G$ is denoted by $|E|$ (or $S(G)$ ) where

$$
S(G)=|E|=\sum_{x y \in E} \frac{1+\sum_{i=1}^{m} p_{i} \circ B(x y)}{2} .
$$

Theorem 26 Two isomorphic m-polar fuzzy graphs $G_{1}=\left(V_{1}, A_{1}, B_{1}\right)$ and $G_{2}=\left(V_{2}, A_{2}, B_{2}\right)$ of the graphs $G_{1}^{*}=\left(V_{1}, E_{1}\right)$ and $G_{2}^{*}=\left(V_{2}, E_{2}\right)$ have same order and size.

Proof Let $\phi$ be an isomorphism from $G_{1}$ onto $G_{2}$. Then $A_{1}(x)=A_{2}(\phi(x))$ for all $x \in V_{1}$ and $p_{i} \circ B_{1}(x y)=p_{i} \circ B_{2}(\phi(x) \phi(y))$ for $i=1,2, \ldots, m, x y \in \widetilde{V_{1}^{2}}$.

Now,

$$
\begin{aligned}
O\left(G_{1}\right) & =\left|V_{1}\right|=\sum_{x \in V_{1}} \frac{1+\sum_{i=1}^{m} p_{i} \circ A_{1}(x)}{2} \\
& =\sum_{\phi(x) \in V_{2}} \frac{1+\sum_{i=1}^{m} p_{i} \circ A_{2}(\phi(x))}{2}=O\left(G_{2}\right)
\end{aligned}
$$

and

$$
\begin{aligned}
S\left(G_{1}\right) & =\left|E_{1}\right|=\sum_{x y \in E_{1}} \frac{1+\sum_{i=1}^{m} p_{i} \circ B_{1}(x y)}{2} \\
& =\sum_{\phi(x) \phi(y) \in E_{2}} \frac{1+\sum_{i=1}^{m} p_{i} \circ B_{2}(\phi(x) \phi(y))}{2}=S\left(G_{2}\right) .
\end{aligned}
$$




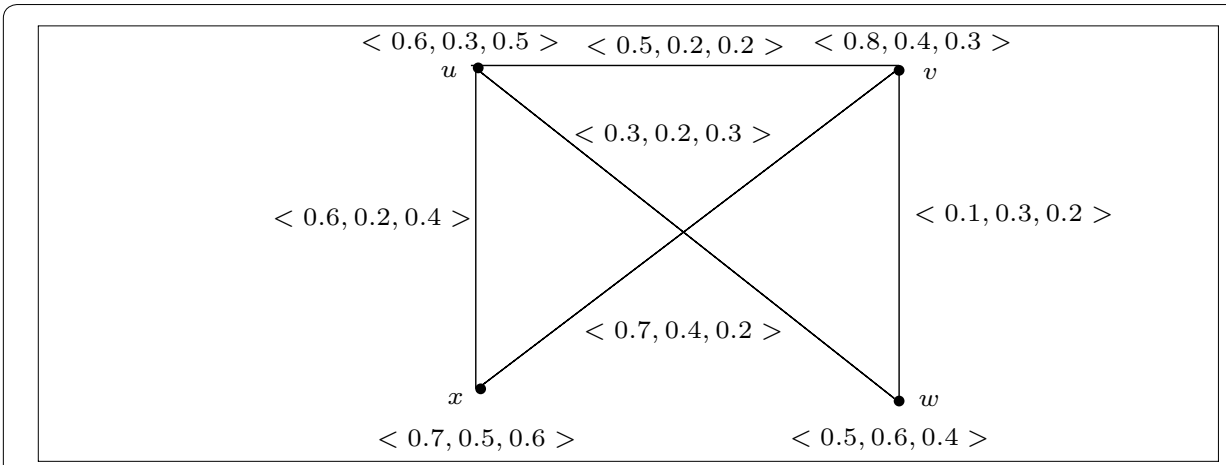

Fig. 5 3-Polar fuzzy graph $G$ and busy value of its vertices

Definition 27 The busy value of a vertex $u$ of an $m$-polar fuzzy graph $G \quad$ is denoted as $D(u)=\left(p_{1} \circ D(u), p_{2} \circ D(u), \ldots, p_{m} \circ D(u)\right)$ where $p_{i} \circ D(u)=\sum_{k} \min \left\{p_{i} \circ A(u), p_{i} \circ A\left(u_{k}\right)\right\} ; u_{k}$ are the neighbors of $u$. The busy value of $G$ is denoted as $D(G)$ where $D(G)=\sum_{k} D\left(u_{k}\right), u_{k} \in V$.

Example 28 Consider the 3-polar fuzzy graph $G=(V, A, B)$ of $G^{*}=(V, E)$ where $\quad V=\{u, v, w, x\}, \quad E=\{u v, v w, u x, u w, v x\}, \quad A=\left\{\frac{\langle 0.6,0.3,0.5\rangle}{u}, \frac{\langle 0.8,0.4,0.3\rangle}{v}\right.$, $\left.\frac{\langle 0.5,0.6,0.4\rangle}{w}, \frac{\langle 0.7,0.5,0.6\rangle}{x}\right\}$ and $B=\left\{\frac{\langle 0.5,0.2,0.2\rangle}{u v}, \frac{\langle 0.1,0.3,0.2\rangle}{v w}, \frac{\langle 0.6,0.2,0.4\rangle}{u x}, \frac{\langle 0.3,0.2,0.3\rangle}{u w}, \frac{\langle 0.7,0.4,0.2\rangle}{v x}\right\}$.

Then we have from Fig. 5 ,

$$
\begin{array}{lll}
p_{1} \circ D(u)=1.7, & p_{2} \circ D(u)=0.9, & p_{3} \circ D(u)=1.2, \\
p_{1} \circ D(v)=1.8, & p_{2} \circ D(v)=1.1, & p_{3} \circ D(v)=0.9, \\
p_{1} \circ D(w)=1, & p_{2} \circ D(w)=0.7, & p_{3} \circ D(w)=0.7, \\
p_{1} \circ D(x)=1.3, & p_{2} \circ D(x)=0.7, & p_{3} \circ D(x)=0.8 .
\end{array}
$$

So, $D(u)=(1.7,0.9,1.2), D(v)=(1.8,1.1,0.9), D(w)=(1,0.7,0.7), D(x)=(1.3,0.7,0.8)$.

Definition 29 If $p_{i} \circ A(u) \leq p_{i} \circ \operatorname{deg}(u)$ for $i=1,2, \ldots, m$, then the vertex $u$ of $G$ is called a busy vertex. Otherwise it is a free vertex.

Definition 30 If $\quad p_{i} \circ B\left(u_{1} v_{1}\right)=\min \left\{p_{i} \circ A\left(u_{1}\right), p_{i} \circ A\left(v_{1}\right)\right\}, \quad i=1,2, \ldots, m \quad$ for $u_{1} v_{1} \in E$, then it is called an effective edge of $G$.

Definition 31 Let $u \in V$ be a vertex of the $m$-polar fuzzy graph $G=(V, A, B)$.

(i) $\quad u$ is called a partial free vertex if it is a free vertex of $G$ and $\bar{G}$.

(ii) $u$ is called a fully free vertex if it is a free vertex of $G$ and it is a busy vertex of $\bar{G}$.

(iii) $u$ is called a partial busy vertex if it is a busy vertex of $G$ and $\bar{G}$.

(iv) $u$ is called a fully busy vertex if it is a busy vertex in $G$ and it is a free vertex of $\bar{G}$.

Theorem 32 Let $\phi$ be an isomorphism from $G_{1}=\left(V_{1}, A_{1}, B_{1}\right)$ onto $G_{2}=\left(V_{2}, A_{2}, B_{2}\right)$. Then $\operatorname{deg}(u)=\operatorname{deg}(\phi(u))$ for all $u \in V_{1}$. 
Proof Since $\phi$ is an isomorphism between $G_{1}$ and $G_{2}$, we have $p_{i} \circ A_{1}(u)=p_{i} \circ A_{2}(\phi(u))$ for all $u \in V_{1}$ and $p_{i} \circ B_{1}\left(x_{1} y_{1}\right)=p_{i} \circ B_{2}\left(\phi\left(x_{1}\right) \phi\left(y_{1}\right)\right)$ for all $x_{1} y_{1} \in \widetilde{V_{1}^{2}}, i=1,2, \ldots, m$.

$$
\text { Hence, } p_{i} \circ \operatorname{deg}(u)=\sum_{\begin{array}{c}
u \neq v \\
u v \in E_{1}
\end{array}} p_{i} \circ B_{1}(u v)=\sum_{\phi(u) \neq \phi(v)} \quad p_{i} \circ B_{2}(\phi(u) \phi(v))=
$$
$p_{i} \circ \operatorname{deg}(\phi(u))$ for $u \in V_{1}, i=1,2, \ldots, m$. So, $\operatorname{deg}(u)=\operatorname{deg}(\phi(u))$ for all $u \in V_{1}$.

Theorem 33 If $\phi$ is an isomorphism from $G_{1}$ onto $G_{2}$ and $u$ is a busy vertex of $G_{1}$, then $\phi(u)$ is a busy vertex of $G_{2}$.

Proof Since $\phi$ is an isomorphism between we have, $p_{i} \circ A_{1}(u)=p_{i} \circ A_{2}(\phi(u)) u \in V_{1}$ and $p_{i} \circ B_{1}\left(x_{1} y_{1}\right)=p_{i} \circ B_{2}\left(\phi\left(x_{1}\right) \phi\left(y_{1}\right)\right)$ for $x_{1} y_{1} \in \widetilde{V_{1}^{2}}, i=1,2, \ldots, m$.

If $u$ is a busy vertex of $G_{1}$, then $p_{i} \circ A_{1}(u) \leq p_{i} \circ \operatorname{deg}(u)$ for $i=1,2, \ldots, m$. Then by the above and Theorem 32, $p_{i} \circ A_{2}(\phi(u))=p_{i} \circ A_{1}(u) \leq p_{i} \circ \operatorname{deg}(u)=p_{i} \circ \operatorname{deg}(\phi(u))$ for $i=1,2, \ldots, m$. Hence, $\phi(u)$ is a busy vertex in $G_{2}$.

Theorem 34 Let the two m-polar fuzzy graphs $G_{1}$ and $G_{2}$ be weak isomorphic. If $u \in V_{1}$ is a busy vertex of $G_{1}$, then the image of $u$ under the weak isomorphism is also busy in $G_{2}$.

Proof Let $\phi: V_{1} \rightarrow V_{2}$ be a weak isomorphism between $G_{1}$ and $G_{2}$.

Then, $p_{i} \circ A_{1}(x)=p_{i} \circ A_{2}(\phi(x))$ for all $x \in V_{1}$ and $p_{i} \circ B_{1}\left(x_{1} y_{1}\right) \leq p_{i} \circ B_{2}\left(\phi\left(x_{1}\right) \phi\left(y_{1}\right)\right)$ for all $x_{1} y_{1} \in \widetilde{V_{1}^{2}}, i=1,2, \ldots, m$.

Let $u \in V_{1}$ be a busy vertex. Then, for $i=1,2, \ldots, m, p_{i} \circ A_{1}(u) \leq p_{i} \circ \operatorname{deg}(u)$.

Now by the above for $i=1,2, \ldots, m$

$$
\begin{aligned}
p_{i} \circ A_{2}(u)= & p_{i} \circ A_{1}(u) \leq p_{i} \circ \operatorname{deg}(u)=\sum_{\begin{array}{c}
u \neq v \\
u v \in E_{1}
\end{array}} p_{i} \circ B_{1}(u v) \\
\leq & \sum_{\substack{\phi(u) \neq \phi(v) \\
\phi(u) \phi(v) \in E_{2}}} p_{i} \circ B_{2}(\phi(u) \phi(v))=p_{i} \circ \operatorname{deg}(\phi(u)) .
\end{aligned}
$$

Hence, $\phi(u)$ is a busy vertex in $G_{2}$.

\section{Complement and isomorphism in $\boldsymbol{m}$-polar fuzzy graphs}

In this section some important properties of isomorphism, weak isomorphism, co weak isomorphism related with complement are discussed.

Theorem 35 Let $G_{1}=\left(V_{1}, A_{1}, B_{1}\right)$ and $G_{2}=\left(V_{2}, A_{2}, B_{2}\right)$ be two m-polar fuzzy graphs of the graphs $G_{1}^{*}=\left(V_{1}, E_{1}\right)$ and $G_{2}^{*}=\left(V_{2}, E_{2}\right)$. If $G_{1} \cong G_{2}$ then $\overline{G_{1}} \cong \overline{G_{2}}$.

Proof Let $G_{1} \cong G_{2}$. Then there exists an isomorphism $\phi: V_{1} \rightarrow V_{2}$ such that $A_{1}(x)=A_{2}(\phi(x))$ for all $x \in V_{1}$ and $p_{i} \circ B_{1}(x y)=p_{i} \circ B_{2}(\phi(x) \phi(y))$, for each $i=1,2, \ldots, m$ and $x y \in \widetilde{V_{1}^{2}}$. 
Now, $\overline{A_{1}}(x)=A_{1}(x)=A_{2}(\phi(x)) \equiv \overline{A_{2}}(\phi(x))$ for all $x \in V_{1}$.

Also, for $i=1,2, \ldots, m$ and $x y \in \widetilde{V}_{1}^{2}$ we have,

$$
\begin{aligned}
p_{i} \circ \overline{B_{1}}(x y) & =\min \left\{p_{i} \circ A_{1}(x), p_{i} \circ A_{1}(y)\right\}-p_{i} \circ B_{1}(x y) \\
& =\min \left\{p _ { i } \circ A _ { 2 } \left(\phi(x), p_{i} \circ A_{2}(\phi(y)\}-p_{i} \circ B_{2}(\phi(x) \phi(y))=p_{i} \circ \overline{B_{2}}(\phi(x) \phi(y)) .\right.\right.
\end{aligned}
$$

Hence, $\phi$ is an isomorphism between $\overline{G_{1}}$ and $\overline{G_{2}}$ i.e., $\overline{G_{1}} \cong \overline{G_{2}}$.

Remark 36 Suppose there is a weak isomorphism between two $m$-polar fuzzy graphs $G_{1}$ and $G_{2}$. Then there may not be a weak isomorphism between $\overline{G_{1}}$ and $\overline{G_{2}}$.

For example, consider two 3-polar fuzzy graphs $G_{1}$ and $G_{2}$ of Fig. 6. Let us now define a mapping $\phi: V_{1} \rightarrow V_{2}$ such that $\phi(a)=u, \phi(b)=v, \phi(c)=w$. Then $\phi$ is a weak isomorphism from $G_{1}$ onto $G_{2}$. But, there is no weak isomorphism from $\overline{G_{1}}$ onto $\overline{G_{2}}$ (see Fig. 7) because $\overline{B_{2}}(u w=\phi(a) \phi(c))=\mathbf{0}=(0,0, \ldots, 0)<\overline{B_{1}}(a c)=(0.1,0.1,0.05)$, and $\overline{B_{2}}(v w=\phi(b) \phi(c))=\mathbf{0}=(0,0, \ldots, 0)<\overline{B_{1}}(b c)=(0.1,0.1,0.1)$.

Remark 37 In a similar way, we can construct example to show that if there is a coweak isomorphism between two $m$-polar fuzzy graphs $G_{1}$ and $G_{2}$ then there may not be a co-weak isomorphism between $\overline{G_{1}}$ and $\overline{G_{2}}$.
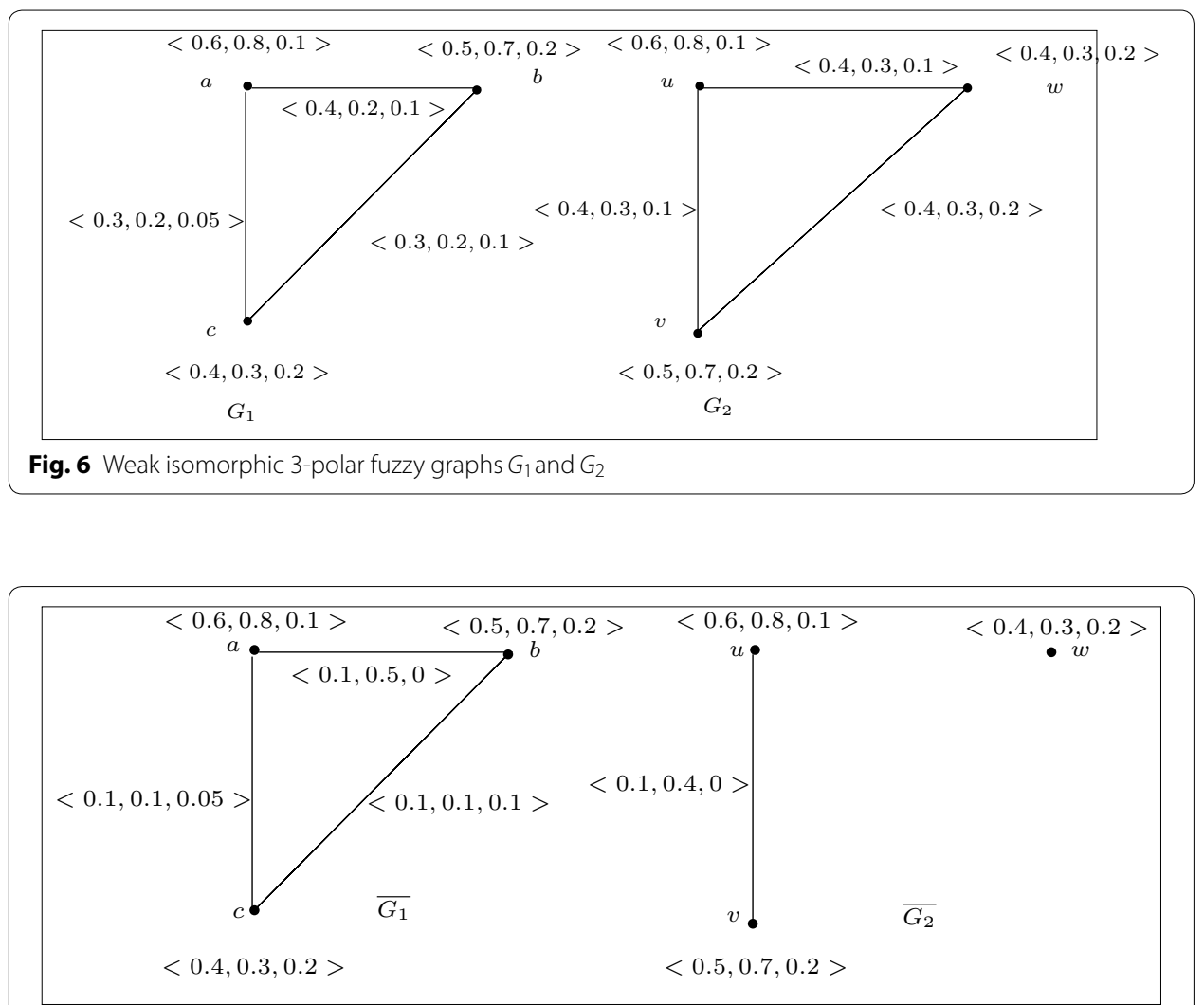

Fig. 7 Example of weak isomorphic graphs whose complement is not weak isomorphic 
Theorem 38 Let $G_{1}=\left(V_{1}, A_{1}, B_{1}\right)$ and $G_{2}=\left(V_{2}, A_{2}, B_{2}\right)$ be two m-polar fuzzy graphs of the graphs $G_{1}^{*}=\left(V_{1}, E_{1}\right)$ and $G_{2}^{*}=\left(V_{2}, E_{2}\right)$ such that $V_{1} \cap V_{2}=\emptyset$. Then $\overline{G_{1}+G_{2}} \cong \overline{G_{1}} \cup \overline{G_{2}}$.

Proof To show that $\overline{G_{1}+G_{2}} \cong \overline{G_{1}} \cup \overline{G_{2}}$, we need to show that there exists an isomorphism between $\overline{G_{1}+G_{2}}$ and $\overline{G_{1}} \cup \overline{G_{2}}$.

We will show that the identity map $I: V_{1} \cup V_{2} \rightarrow V_{1} \cup V_{2}$ is the required isomorphism between them. For this, we will show the following:

for all $x \in V_{1} \cup V_{2}, \overline{\left(A_{1}+A_{2}\right)}(x)=\left(\overline{A_{1}} \cup \overline{A_{2}}\right)(x)$,

and $p_{i} \circ \overline{\left(B_{1}+B_{2}\right)}(x y)=p_{i} \circ\left(\overline{B_{1}} \cup \overline{B_{2}}\right)(x y)$ for $i=1,2, \ldots, m$ and $x y \in \widehat{V_{1} \times V_{2}}{ }^{2}$.

Let $x \in V_{1} \cup V_{2}$.

Then

$$
\begin{aligned}
\overline{\left(A_{1}+A_{2}\right)}(x) & =\left(A_{1}+A_{2}\right)(x)=\left(A_{1} \cup A_{2}\right)(x) \quad \text { (by Definition 8) } \\
& = \begin{cases}A_{1}(x) & \text { if } x \in V_{1}-V_{2} \\
A_{2}(x) & \text { if } x \in V_{2}-V_{1}\end{cases} \\
& =\left\{\begin{array}{ll}
\overline{A_{1}}(x) & \text { if } x \in V_{1}-V_{2} \\
\overline{A_{2}}(x) & \text { if } x \in V_{2}-V_{1}
\end{array}=\left(\overline{A_{1}} \cup \overline{A_{2}}\right)(x) .\right.
\end{aligned}
$$

Now for each $i=1,2, \ldots, m$ and $x y \in{\widetilde{V_{1} \times V_{2}^{2}}}^{2}$ we have,

$$
\begin{aligned}
p_{i} \circ & \overline{\left(B_{1}+B_{2}\right)}(x y) \\
& =\min \left\{p_{i} \circ\left(A_{1}+A_{2}\right)(x), p_{i} \circ\left(A_{1}+A_{2}\right)(y)\right\}-p_{i} \circ\left(B_{1}+B_{2}\right)(x y) \\
& = \begin{cases}\min \left\{p_{i} \circ\left(A_{1} \cup A_{2}\right)(x), p_{i} \circ\left(A_{1} \cup A_{2}\right)(y)\right\}-p_{i} \circ\left(B_{1} \cup B_{2}\right)(x y), & \text { if } x y \in E_{1} \cup E_{2} \\
\min \left\{p_{i} \circ\left(A_{1} \cup A_{2}\right)(x), p_{i} \circ\left(A_{1} \cup A_{2}\right)(y)\right\}-\min \left\{p_{i} \circ A_{1}(x), p_{i} \circ A_{2}(y)\right\}, & \text { if } x y \in E^{\prime}\end{cases} \\
& = \begin{cases}\min \left\{p_{i} \circ A_{1}(x), p_{i} \circ A_{1}(y)\right\}-p_{i} \circ B_{1}(x y), & \text { if } x y \in E_{1}-E_{2} \\
\min \left\{p_{i} \circ A_{2}(x), p_{i} \circ A_{2}(y)\right\}-p_{i} \circ B_{2}(x y), & \text { if } x y \in E_{2}-E_{1} \\
\min \left\{p_{i} \circ A_{1}(x), p_{i} \circ A_{2}(y)\right\}-\min \left\{p_{i} \circ\left(A_{1}\right)(x), p_{i} \circ\left(A_{2}\right)(y)\right\}, & \text { if } x y \in E^{\prime}\end{cases} \\
& = \begin{cases}p_{i} \circ \overline{B_{1}}(x y), & \text { if } x y \in E_{1}-E_{2} \\
p_{i} \circ \overline{B_{2}}(x y), & \text { if } x y \in E_{2}-E_{1} \\
0, & \text { if } x y \in E^{\prime}\end{cases} \\
& =p_{i} \circ\left(\overline{B_{1}} \cup \overline{B_{2}}\right)(x y) .
\end{aligned}
$$

Theorem 39 Let $G_{1}=\left(V_{1}, A_{1}, B_{1}\right)$ and $G_{2}=\left(V_{2}, A_{2}, B_{2}\right)$ be two m-polar fuzzy graphs of the graphs $G_{1}^{*}=\left(V_{1}, E_{1}\right)$ and $G_{2}^{*}=\left(V_{2}, E_{2}\right)$ such that $V_{1} \cap V_{2}=\emptyset$. Then $\overline{G_{1} \cup G_{2}} \cong \overline{G_{1}}+\overline{G_{2}}$.

Proof Consider the identity map $I: V_{1} \cup V_{2} \rightarrow V_{1} \cup V_{2}$. We will show that $I$ is the required isomorphism between $\overline{G_{1} \cup G_{2}}$ and $\overline{G_{1}}+\overline{G_{2}}$.

For this, we will show the following:

for all $x \in V_{1} \cup V_{2}, \overline{\left(A_{1} \cup A_{2}\right)}(x)=\left(\overline{A_{1}}+\overline{A_{2}}\right)(x)$,

and $p_{i} \circ \overline{\left(B_{1} \cup B_{2}\right)}(x y)=p_{i} \circ\left(\overline{B_{1}}+\overline{B_{2}}\right)(x y)$ for $i=1,2, \ldots, m$ and $x y \in \widehat{V_{1} \times V_{2}}{ }^{2}$.

Let $x \in V_{1} \cup V_{2}$.

Then 


$$
\begin{aligned}
\overline{A_{1} \cup A_{2}}(x) & =\left(A_{1} \cup A_{2}\right)(x) \\
& = \begin{cases}A_{1}(x), & \text { if } x \in V_{1}-V_{2} \\
A_{2}(x), & \text { if } x \in V_{2}-V_{1}\end{cases} \\
& = \begin{cases}\overline{A_{1}}(x), & \text { if } x \in V_{1}-V_{2} \\
\overline{A_{2}}(x), & \text { if } x \in V_{2}-V_{1}\end{cases} \\
& =\left(\overline{A_{1}} \cup \overline{A_{2}}\right)(x)
\end{aligned}
$$

and for $i=1,2, \ldots, m, x y \in{\widetilde{V_{1} \times V_{2}}}^{2}$ we have,

$$
\begin{aligned}
& p_{i} \circ \overline{\left(B_{1} \cup B_{2}\right)}(x y) \\
& =\min \left\{p_{i} \circ\left(A_{1} \cup A_{2}\right)(x), p_{i} \circ\left(A_{1} \cup A_{2}\right)(y)\right\}-p_{i} \circ\left(B_{1} \cup B_{2}\right)(x y) \\
& = \begin{cases}\min \left\{p_{i} \circ A_{1}(x), p_{i} \circ A_{1}(y)\right\}-p_{i} \circ B_{1}(x y), & \text { if } x y \in E_{1}-E_{2} \\
\min \left\{p_{i} \circ A_{2}(x), p_{i} \circ A_{2}(y)\right\}-p_{i} \circ B_{2}(x y), & \text { if } x y \in E_{2}-E_{1} \\
\min \left\{p_{i} \circ A_{1}(x), p_{i} \circ A_{2}(y)\right\}-0, & \text { if } x \in V_{1}, y \in V_{2}\end{cases} \\
& = \begin{cases}p_{i} \circ \overline{B_{1}}(x y), & \text { if } x y \in E_{1}-E_{2} \\
p_{i} \circ \overline{B_{2}}(x y), & \text { if } x y \in E_{2}-E_{1} \\
\min \left\{p_{i} \circ A_{1}(x), p_{i} \circ A_{2}(y)\right\}-0, & \text { if } x \in V_{1}, y \in V_{2}\end{cases} \\
& = \begin{cases}p_{i} \circ \overline{B_{1}}(x y), & \text { if } x y \in E_{1}-E_{2} \\
p_{i} \circ \overline{B_{2}}(x y), & \text { if } x y \in E_{2}-E_{1} \\
\min \left\{p_{i} \circ A_{1}(x), p_{i} \circ A_{2}(y)\right\}-0, & \text { if } x y \in E^{\prime}\end{cases} \\
& =p_{i} \circ\left(\overline{B_{1}}+\overline{B_{2}}\right)(x y) \text {. }
\end{aligned}
$$

This completes the proof.

Theorem 40 Let $G_{1}=\left(V_{1}, A_{1}, B_{1}\right)$ and $G_{2}=\left(V_{2}, A_{2}, B_{2}\right)$ be two strong m-polar fuzzy graphs of the graphs $G_{1}^{*}=\left(V_{1}, E_{1}\right)$ and $G_{2}^{*}=\left(V_{2}, E_{2}\right)$ respectively. Then $\overline{G_{1} \circ G_{2}} \cong \overline{G_{1}} \circ \overline{G_{2}}$.

Proof Let $G_{1} \circ G_{2}=\left(V_{1} \times V_{2}, A_{1} \circ A_{2}, B_{1} \circ B_{2}\right)$ be an $m$-polar fuzzy graph of the graph $G^{*}=(V, E)$ where $V=V_{1} \times V_{2}$ and $E=\left\{\left(x, x_{2}\right)\left(x, y_{2}\right): x \in V_{1}, x_{2} y_{2} \in E_{2}\right\} \cup\left\{\left(x_{1}, z\right)\left(y_{1}, z\right)\right.$ : $\left.z \in V_{2}, x_{1} y_{1} \in E_{1}\right\} \cup\left\{\left(x_{1}, x_{2}\right)\left(y_{1}, y_{2}\right): x_{1} y_{1} \in E_{1}, x_{2} \neq y_{2}\right\}$.

We show that the identity map $I$ is the required isomorphism between the graphs $\overline{G_{1} \circ G_{2}}$ and $\overline{G_{1}} \circ \overline{G_{2}}$. Let us consider the identity map $I: V_{1} \times V_{2} \rightarrow V_{1} \times V_{2}$.

In order to show that $I$ is the required isomorphism, we show that for each $i=1,2, \ldots, m$ and for all $x y \in \widetilde{V_{1} \times V_{2}^{2}}, p_{i} \circ \overline{\left(B_{1} \circ B_{2}\right)}(x y)=p_{i} \circ\left(\overline{B_{1}} \circ \overline{B_{2}}\right)(x y)$. Several cases may arise.

Case $(i)$ : Let $e=\left(x, x_{2}\right)\left(x, y_{2}\right)$ where $x \in V_{1}, x_{2} y_{2} \in E_{2}$. Then $e \in E$.

Since $G_{1} \circ G_{2}$ is strong $m$-polar fuzzy graph, we have for each $i=1,2, \ldots, m$

$$
\begin{aligned}
& p_{i} \circ \overline{\left(B_{1} \circ B_{2}\right)}(e)=0 \text { and } \\
& p_{i} \circ\left(\overline{B_{1}} \circ \overline{B_{2}}\right)(e)=\min \left\{p_{i} \circ A_{1}(x), p_{i} \circ \overline{B_{2}}\left(x_{2} y_{2}\right)\right\}=0
\end{aligned}
$$

(since $G_{2}$ is strong and $x_{2} y_{2} \in E_{2}$, therefore for each $i=1,2, \ldots, m, p_{i} \circ \overline{B_{2}}\left(x_{2} y_{2}\right)=0$ ).

Case (ii): Let $e=\left(x, x_{2}\right)\left(x, y_{2}\right)$ where $x_{2} \neq y_{2}, x_{2} y_{2} \notin E_{2}$. Then $e \notin E$. 
So for each $i=1,2, \ldots, m, p_{i} \circ\left(B_{1} \circ B_{2}\right)(e)=0$ and

$$
\begin{aligned}
p_{i} \circ \overline{\left(B_{1} \circ B_{2}\right)}(e) & =\min \left\{p_{i} \circ\left(A_{1} \circ A_{2}\right)\left(x, x_{2}\right), p_{i} \circ\left(A_{1} \circ A_{2}\right)\left(x, y_{2}\right)\right\} \\
& =\min \left\{p_{i} \circ A_{1}(x), p_{i} \circ A_{2}\left(x_{2}\right), p_{i} \circ A_{2}\left(y_{2}\right)\right\} .
\end{aligned}
$$

Again, since $x_{2} y_{2} \in \overline{E_{2}}$, therefore for each $i=1,2, \ldots, m$,

$$
\begin{aligned}
p_{i} \circ\left(\overline{B_{1}} \circ \overline{B_{2}}\right)(e) & =\min \left\{p_{i} \circ A_{1}(x), p_{i} \circ \overline{B_{2}}\left(x_{2} y_{2}\right)\right\} \\
& =\min \left\{p_{i} \circ A_{1}(x), p_{i} \circ A_{2}\left(x_{2}\right), p_{i} \circ A_{2}\left(y_{2}\right)\right\}
\end{aligned}
$$

Case (iii): Let $e=\left(x_{1}, z\right)\left(y_{1}, z\right)$ where $x_{1} y_{1} \in E_{1}, z \in V_{2}$.

Then $e \in E$. So for each $i=1,2, \ldots, m, p_{i} \circ \overline{\left(B_{1} \circ B_{2}\right)}(e)=0$ as in Case (i).

Also, since $x_{1} y_{1} \notin \overline{E_{1}}$, therefore for each $i=1,2, \ldots, m, p_{i} \circ\left(\overline{B_{1}} \circ \overline{B_{2}}\right)(e)=0$.

Case (iv): Let $e=\left(x_{1}, z\right)\left(y_{1}, z\right)$ where $x_{1} y_{1} \notin E_{1}, z \in V_{2}$. Then $e \notin E$.

Hence for each $i=1,2, \ldots, m, p_{i} \circ\left(B_{1} \circ B_{2}\right)(e)=0$,

$$
\begin{aligned}
p_{i} \circ \overline{\left(B_{1} \circ B_{2}\right)}(e) & =\min \left\{p_{i} \circ\left(A_{1} \circ A_{2}\right)\left(x_{1}, z\right), p_{i} \circ\left(A_{1} \circ A_{2}\right)\left(y_{1}, z\right)\right\} \\
& =\min \left\{p_{i} \circ A_{1}\left(x_{1}\right), p_{i} \circ A_{1}\left(y_{1}\right), p_{i} \circ A_{2}(z)\right\} \text { and } \\
p_{i} \circ \overline{\left(B_{1} \circ \overline{B_{2}}\right)(e)} & =\min \left\{p_{i} \circ A_{2}(z), p_{i} \circ \overline{B_{1}}\left(x_{1} y_{1}\right)\right\} \\
& =\min \left\{p_{i} \circ A_{1}\left(x_{1}\right), p_{i} \circ A_{1}\left(y_{1}\right), p_{i} \circ A_{2}(z)\right\}\left(G_{1}\right. \text { being strong). }
\end{aligned}
$$

Case $(v)$ : Let $e=\left(x_{1}, x_{2}\right)\left(y_{1}, y_{2}\right)$ where $x_{1} y_{1} \in E_{1}, x_{2} \neq y_{2}$. Then $e \in E$. So we have for each $i=1,2, \ldots, m, p_{i} \circ \overline{\left(B_{1} \circ B_{2}\right)}(e)=0$ as in Case (i).

Also, since $x_{1} y_{1} \in E_{1}$, we have for each $i=1,2, \ldots, m, p_{i} \circ\left(\overline{B_{1}} \circ \overline{B_{2}}\right)(e)=0$.

Case $(v i)$ : Let $e=\left(x_{1}, x_{2}\right)\left(y_{1}, y_{2}\right)$ where $x_{1} y_{1} \notin E_{1}, x_{2} \neq y_{2}$. Then $e \notin E$ and hence for each $i=1,2, \ldots, m, p_{i} \circ\left(B_{1} \circ B_{2}\right)(e)=0$,

$$
\begin{aligned}
p_{i} \circ \overline{\left(B_{1} \circ B_{2}\right)}(e) & =\min \left\{p_{i} \circ\left(A_{1} \circ A_{2}\right)\left(x_{1}, x_{2}\right), p_{i} \circ\left(A_{1} \circ A_{2}\right)\left(y_{1}, y_{2}\right)\right\} \\
& =\min \left\{p_{i} \circ A_{1}\left(x_{1}\right), p_{i} \circ A_{1}\left(y_{1}\right), p_{i} \circ A_{2}\left(x_{2}\right), p_{i} \circ A_{2}\left(y_{2}\right)\right\}
\end{aligned}
$$

and since $x_{1} y_{1} \in \overline{E_{1}}$,

$$
\begin{aligned}
p_{i} \circ\left(\overline{B_{1}} \circ \overline{B_{2}}\right)(e) & =\min \left\{p_{i} \circ A_{2}\left(x_{2}\right), p_{i} \circ A_{2}\left(y_{2}\right), p_{i} \circ \overline{B_{1}}\left(x_{1} y_{1}\right)\right\} \\
& =\min \left\{p_{i} \circ A_{1}\left(x_{1}\right), p_{i} \circ A_{1}\left(y_{1}\right), p_{i} \circ A_{2}\left(x_{2}\right), p_{i} \circ A_{2}\left(y_{2}\right)\right\}\left(\overline{G_{1}} \text { being strong by [10] }\right)
\end{aligned}
$$

Case (vii): Finally, let $e=\left(x_{1}, x_{2}\right)\left(y_{1}, y_{2}\right)$ where $x_{1} y_{1} \notin E_{1}, x_{2} y_{2} \notin E_{2}$. Then $e \notin E$ and hence for each $i=1,2, \ldots, m, p_{i} \circ\left(B_{1} \circ B_{2}\right)(e)=0$,

$$
p_{i} \circ \overline{\left(B_{1} \circ B_{2}\right)}(e)=\min \left\{p_{i} \circ\left(A_{1} \circ A_{2}\right)\left(x_{1}, x_{2}\right), p_{i} \circ\left(A_{1} \circ A_{2}\right)\left(y_{1}, y_{2}\right)\right\} .
$$

Now, $x_{1} y_{1} \in \overline{E_{1}}$ and if $x_{2}=y_{2}=z$, then we have the Case (iv).

Again, if $x_{1} y_{1} \in \overline{E_{1}}$ and if $x_{2} \neq y_{2}$, then we have Case (vi).

Thus combining all the cases we have, for each $i=1,2, \ldots, m$, and $x y \in \widehat{V_{1} \times V_{2}}$

$$
p_{i} \circ \overline{\left(B_{1} \circ B_{2}\right)}(x y)=p_{i} \circ\left(\overline{B_{1}} \circ \overline{B_{2}}\right)(x y) \text {. }
$$


Remark 41 If $G_{1}$ and $G_{2}$ are not strong, then $\overline{G_{1} \circ G_{2}} \supsetneqq \overline{G_{1}} \circ \overline{G_{2}}$ always. For example, consider the two 3-polar fuzzy graphs $G_{1}$ and $G_{2}$ which are not strong (see Fig. 8). From Figs. 8 and 9, we see that, $\overline{G_{1} \circ G_{2}} ¥ \overline{G_{1}} \circ \overline{G_{2}}$.

\section{Applications}

Now a days, fuzzy graphs and bipolar fuzzy graphs are most familiar graphs to us and they can also be thought of as 1-polar and 2-polar fuzzy graphs respectively. These graphs have many important application in social networks, medical diagnosis, computer networks, database theory, expert system, neural networks, artificial intelligence, signal
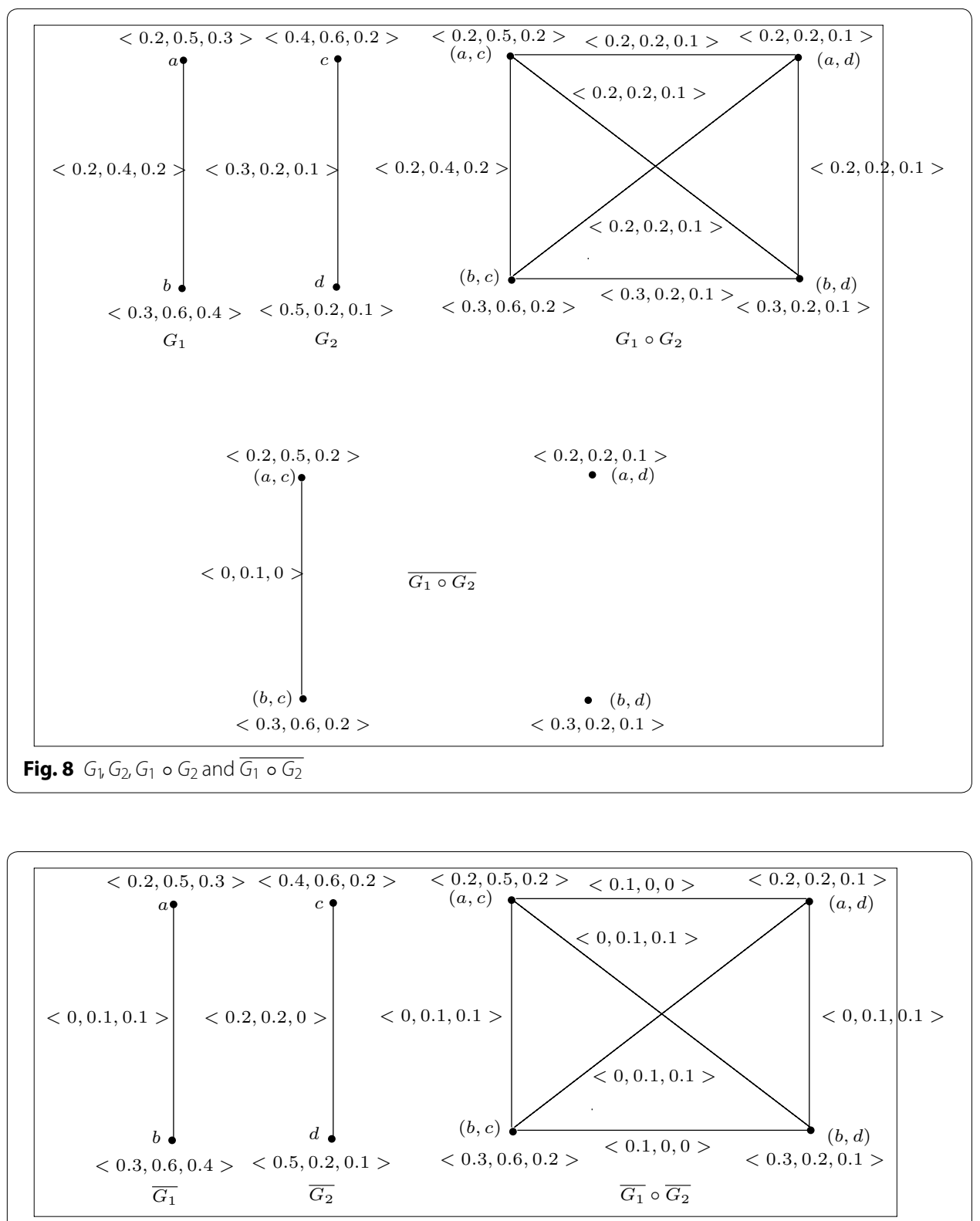

Fig. 9 Example of 3-polar fuzzy graphs $G_{1}$ and $G_{2}$ where $\overline{G_{1} \circ G_{2}} \not \overline{G_{1}} \circ \overline{G_{2}}$ 
processing, pattern recognition, engineering science, cluster analysis, etc. The concepts of bipolar fuzzy graphs can be generalized to $m$-polar fuzzy graphs. For example, consider the sorting of mangoes and guavas. Now the different characteristics of a given fruit can change the decision in sorting process more towards the decision mango or vice versa. There are two poles present in this case. One is 100\% sure mango and the other is 100\% sure guava. This shows that the situation is bipolar. This situation can be generalized further by adding a new fruit, for example sweet lemon into the sorting process.

\section{Graphical representation of tug of war}

Consider the another example of tug of war where two people pull the rope in opposite directions. Here, who uses the bigger force, the center of the rope will move in the respective direction of their pulling. The situation is symmetric in this case. We present an example where $m$ people pull a special rope in $m$ different directions. We use this example to represent it as an $m$-polar fuzzy graph. We assume that $O$ is the origin and there are $m$ straight paths leading from $O$. We also assume that there is a wall in between these paths. In this setting, we have the special rope with one node at $O$ and $m$ endings going out from this nodes-one end corresponding to each of the paths. Suppose on every path there is a man standing and pulling the rope in the direction of the path on which he is standing. This situation can be represented as an $m$-polar fuzzy graph by considering the nodes as $m$-polar fuzzy set and edges between them as $m$-polar fuzzy relations, which is shown in Fig. 10. In this context, one can ask the question what is the strength require in order to pull the node $O$ from the center into one of the paths (assuming no friction)? The answer to this is that if the corresponding forces which are pulling the rope are $F_{k}, k=1,2, \ldots, m$, then the node $O$ will move to the $j$ th path if $F_{j}>\sum_{k=1,2, \ldots, m} F_{k}$.

\section{Evaluation graph corresponding to the teacher's evaluation by the students}

In this section we present the model of $m$-polar fuzzy graph which is used in evaluating the teachers by the students of 4th semester of a department in an university during the session 2015-2016. Here the nodes represent the teachers of the corresponding department and edges represent the relationship between two teachers. Suppose the department has six teachers denoted as $T=\left\{t_{1}, t_{2}, t_{3}, t_{4}, t_{5}, t_{6}\right\}$. The membership value of each node represents the corresponding teachers feedback response of the students depending

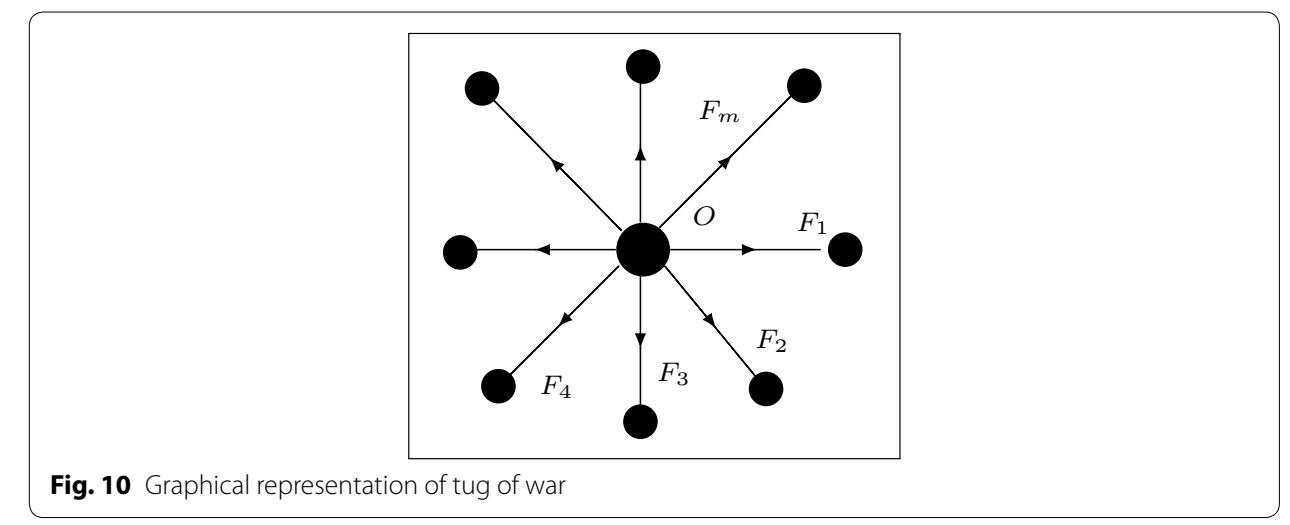


on the following: \{regularity of classes, style of presentation, quality of lectures, generation of interest and encouraging future reading among students, updated information\}. Since all the above characteristics of a teacher according to the different students are uncertain in real life, therefore we consider 5-polar fuzzy subset of the vertex set $T$ (Fig. 11).

In the Table 1, the membership values of the teacher's are given which is according to the evaluation of the students.

Edge membership values which represent the relationship between the teachers can be calculated by using the relation $p_{i} \circ B(u v) \leq \min \left\{p_{i} \circ A(u), p_{i} \circ A(v)\right\}$ for all $u, v \in T$, $i=1,2, \ldots, 5$. These values are given in the Table 2 .

We rank the teacher's performance according the following:

Teacher's average response score $<60 \%$, teacher's performance according to the students is Average.

Teacher's average response score $\geq 60 \%$ and $<70 \%$, teacher's performance according to the students is Good.

Teacher's average response score $\geq 70 \%$ and $<80 \%$, teacher's performance according to the students is Very Good.

Teacher's average response score is $\geq 80 \%$, teacher's performance according to the students is Excellent.

From the Table 3 , we see that the performance of the teachers $t_{1}, t_{2}, t_{5}, t_{6}$ are very good whereas the performance of the teachers $t_{3}$ and $t_{4}$ are excellent. Among these teachers, teacher $t_{3}$ is the best teacher according the response score of the students of the department during the session 2015-2016.

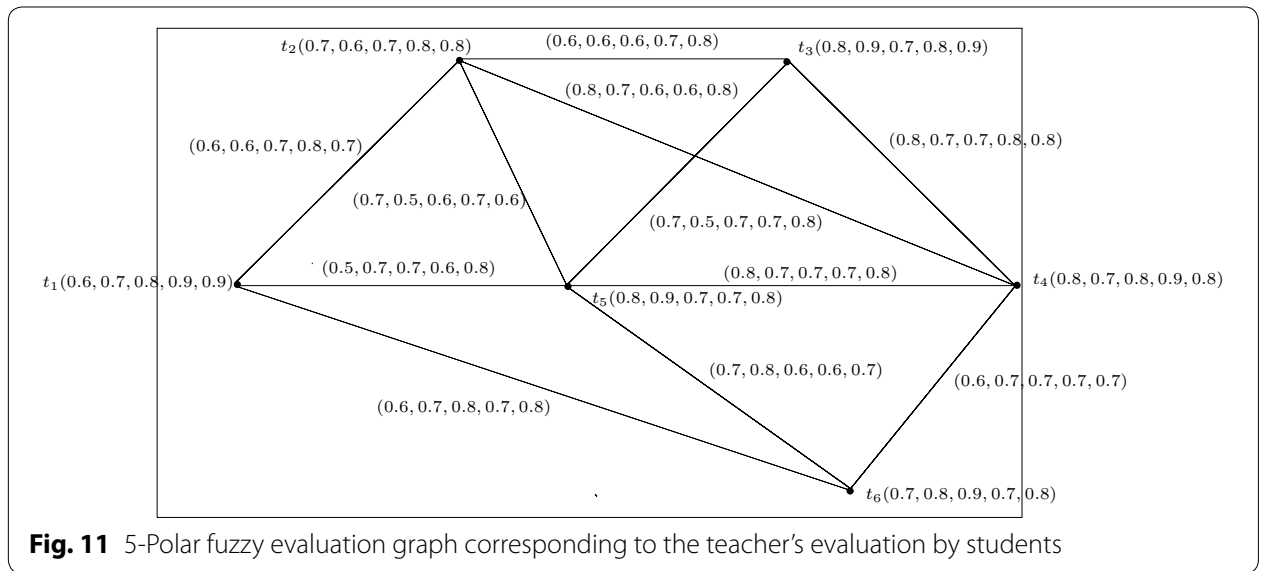

Table 1 5-Polar fuzzy set $A$ of $T$

\begin{tabular}{lllllll}
\hline & $\boldsymbol{t}_{\mathbf{1}}$ & $\boldsymbol{t}_{\mathbf{2}}$ & $\boldsymbol{t}_{\mathbf{3}}$ & $\boldsymbol{t}_{\mathbf{4}}$ & $\boldsymbol{t}_{\mathbf{5}}$ & $\boldsymbol{t}_{\mathbf{6}}$ \\
\hline$p_{1} \circ A$ & 0.6 & 0.7 & 0.8 & 0.8 & 0.8 & 0.7 \\
$p_{2} \circ A$ & 0.7 & 0.6 & 0.9 & 0.7 & 0.9 & 0.8 \\
$p_{3} \circ A$ & 0.8 & 0.7 & 0.7 & 0.8 & 0.7 & 0.9 \\
$p_{4} \circ A$ & 0.9 & 0.8 & 0.8 & 0.9 & 0.7 & 0.7 \\
$p_{5} \circ A$ & 0.9 & 0.8 & 0.9 & 0.8 & 0.8 & 0.8 \\
\hline
\end{tabular}


Table 2 5-Polar fuzzy relation $B$ on $A$

\begin{tabular}{llllllllllll}
\hline & $\boldsymbol{t}_{\mathbf{1}} \boldsymbol{t}_{\mathbf{2}}$ & $\boldsymbol{t}_{\mathbf{1}} \boldsymbol{t}_{\mathbf{5}}$ & $\boldsymbol{t}_{\mathbf{1}} \boldsymbol{t}_{\mathbf{6}}$ & $\boldsymbol{t}_{\mathbf{2}} \boldsymbol{t}_{\mathbf{3}}$ & $\boldsymbol{t}_{\mathbf{2}} \boldsymbol{t}_{\mathbf{4}}$ & $\boldsymbol{t}_{\mathbf{2}} \boldsymbol{t}_{\mathbf{5}}$ & $\boldsymbol{t}_{\mathbf{3}} \boldsymbol{t}_{\mathbf{4}}$ & $\boldsymbol{t}_{\mathbf{3}} \boldsymbol{t}_{\mathbf{5}}$ & $\boldsymbol{t}_{\mathbf{4}} \boldsymbol{t}_{\mathbf{5}}$ & $\boldsymbol{t}_{\mathbf{4}} \boldsymbol{t}_{\mathbf{6}}$ & $\boldsymbol{t}_{\mathbf{5}} \boldsymbol{t}_{\mathbf{6}}$ \\
\hline$p_{1} \circ A$ & 0.6 & 0.5 & 0.6 & 0.6 & 0.8 & 0.7 & 0.8 & 0.7 & 0.8 & 0.6 & 0.7 \\
$p_{2} \circ A$ & 0.6 & 0.7 & 0.7 & 0.6 & 0.7 & 0.5 & 0.7 & 0.5 & 0.7 & 0.7 & 0.8 \\
$p_{3} \circ A$ & 0.7 & 0.7 & 0.8 & 0.6 & 0.6 & 0.6 & 0.7 & 0.7 & 0.7 & 0.7 & 0.6 \\
$p_{4} \circ A$ & 0.8 & 0.6 & 0.7 & 0.7 & 0.6 & 0.7 & 0.8 & 0.7 & 0.7 & 0.7 & 0.6 \\
$p_{5} \circ A$ & 0.7 & 0.8 & 0.8 & 0.8 & 0.8 & 0.6 & 0.8 & 0.8 & 0.8 & 0.7 & 0.7 \\
\hline
\end{tabular}

Table 3 Average response score of the teachers

\begin{tabular}{lllllll}
\hline & Teachers & & & & \\
\cline { 2 - 7 } & $\boldsymbol{t}_{\mathbf{1}}$ & $\boldsymbol{t}_{\mathbf{2}}$ & $\boldsymbol{t}_{\mathbf{3}}$ & $\boldsymbol{t}_{\mathbf{4}}$ & $\boldsymbol{t}_{\mathbf{5}}$ & $\boldsymbol{t}_{\mathbf{6}}$ \\
\hline Scores & 0.78 & 0.72 & 0.82 & 0.8 & 0.78 & 0.78 \\
\hline
\end{tabular}

\section{Conclusions}

The theory of fuzzy graphs play an important role in many fields including decision makings, computer networking and management sciences. An m-polar fuzzy graph can be used to represent real world problems which involve multi-agent, multi-attribute, multiobject, multi-index, multi-polar information and uncertainty. In this research paper, we have studied the isomorphic properties of $m$-polar fuzzy graphs with some applications. We are extending our research work on $m$-polar fuzzy intersection graphs, $m$-polar fuzzy interval graphs, properties of $m$-polar fuzzy hypergraphs, degrees of vertices of $m$-polar fuzzy graphs and its application in decision making, etc.

Authors' contributions

Both authors have significant contributions to this paper and the final form of this paper is approved by both of them. Both authors read and approved the final manuscript.

\section{Acknowledgements}

The authors would wish to express their sincere gratitude to the Editor in Chief and anonymous referees for their valuable comments and helpful suggestions.

Competing interests

The authors declare that they have no competing interests.

Received: 14 July 2016 Accepted: 1 December 2016

Published online: 20 December 2016

\section{References}

Akram M (2011) Bipolar fuzzy grpahs. Inf Sci 181(24):5548-5564

Akram M (2013) Bipolar fuzzy graphs with applications. Knowl Based Syst 39:1-8

Akram M, Younas HR (2015) Certain types of irregular m-polar fuzzy graphs. J Appl Math Comput. doi:10.1007/ s12190-015-0972-9

Akram M, Akmal R, Alsheri N (2016) On m-polar fuzzy graph structures. Springerplus 5:1448, doi:10.1186/ s40064-016-3066-8

Al-Hawary T (2011) Complete fuzzy graphs. Int J Math Combin 4:26-34

Bhutani KR (1989) On automorphism of fuzzy graphs. Pattern Recognit Lett 9:159-162

Bhutani KR, Moderson J, Rosenfeld A (2004) On degrees of end nodes and cut nodes in fuzzy graphs. Iran J Fuzzy Syst 1(1):57-64

Chen J, Li S, Ma S, Wang X (2014) rm-polar fuzzy sets: an extension of bipolar fuzzy sets. Sci World J. doi:10.1155/2014/416530

Ghorai G, Pal M (2015a) On some operations and density of m-polar fuzzy graphs. Pac Sci Rev A Nat Sci Eng 17(1):14-22 Ghorai G, Pal M (2015b) Ceratin types of product bipolar fuzzy graphs. Int J Appl Comput Math. doi:10.1007/ s40819-015-0112-0 
Ghorai G, Pal M (2016a) Some properties of m-polar fuzzy graphs. Pac Sci Rev A Nat Sci Eng. 18(1):38-46. doi:10.1016/j. psra.2016.06.004

Ghorai G, Pal M (2016b) A study on m-polar fuzzy planar graphs. Int J Comput Sci Math 7(3):283-292

Ghorai G, Pal M (2016c) Faces and dual of m-polar fuzzy planar graphs. J Intell Fuzzy Syst 31 (3):2043-2049

Harary F (1972) Graph theory, 3rd edn. Addison-Wesley, Reading

Koczy LT (1992) Fuzzy graphs in the evaluation and optimization of networks. Fuzzy Sets Syst 46:307-319

Lee KM (2000) Bipolar valued fuzzy sets and their basic operations. In: Proceedings of the international conference, Bangkok, Thailand, pp 307-317

Lee-kwang H, Lee KM (1995) Fuzzy hypergraph and fuzzy partition. IEEE Trans Syst Man Cybernet 25:196-201

Mordeson JN, Peng CS (1994) Operations on fuzzy graphs. Inf Sci 19:159-170

Mordeson JN, Nair PS (2000) Fuzzy graphs and hypergraphs. Physica Verlag, Heidelberg

Nagoorgani A, Radha K (2008) On regular fuzzy graphs. J Phys Sci 12:33-40

Rosenfeld A (1975) Fuzzy graphs. In: Zadeh LA, Fu KS, Shimura M (eds) Fuzzy sets and their applications. Academic Press, New York, pp 77-95

Rashmanlou H, Samanta S, Pal M, Borzooei RA (2015a) A study on bipolar fuzzy graphs. J Intell Fuzzy Syst 28:571-580 Rashmanlou H, Samanta S, Pal M, Borzooei RA (2015b) Bipolar fuzzy graphs with categorical properties. Int I Comput Intell Syst 8(5):808-818

Rashmanlou H, Samanta S, Pal M, Borzooei RA (2016) Product of bipolar fuzzy graphs and their degree. Int I Gen Syst 45(1):1-14

Samanta S, Pal M (2011a) Fuzzy tolerance graphs. Int J Latest Trends Math 1(2):57-67

Samanta S, Pal M (2011 b) Fuzzy threshold graphs. CIIT Int J Fuzzy Syst 3(12):360-364

Samanta S, Pal M (2012a) Bipolar fuzzy hypergraphs. Int J Fuzzy Logic Syst 2(1):17-28

Samanta S, Pal M (2012b) Irregular bipolar fuzzy graphs. Int J Appl Fuzzy Sets 2:91-102

Samanta S, Pal M (2013) Fuzzy k-competition graphs and p-competitions fuzzy graphs. Fuzzy Inf Eng 5(2):191-204

Samanta S, Pal M (2014) Some more results on bipolar fuzzy sets and bipolar fuzzy intersection graphs. J Fuzzy Math 22(2): $1-10$

Samanta S, Pal M (2015) Fuzzy planar graphs. IEEE Trans Fuzzy Syst 23(6):1936-1942

Sunitha MS, Vijayakumar (2002) A Complement of fuzzy graphs. Indian J Pure Appl Math 33:1451-1464

Yang HL, Li SG, Yang WH, Lu Y (2013) Notes on "bipolar fuzzy graphs". Inf Sci 242:113-121

Zadeh LA (1965) Fuzzy sets. Inf Control 8:338-353

Zhang WR (1994) Bipolar fuzzy sets and relations: a computational framework for cognitive modeling and multiagent decision analysis. In: Proceedings of IEEE conference, pp 305-309

Zhang WR (1998) Bipolar fuzzy sets. In: Proceedings of Fuzzy-IEEE, pp 835-840

\section{Submit your manuscript to a SpringerOpen ${ }^{\circ}$ journal and benefit from:}

- Convenient online submission

- Rigorous peer review

- Immediate publication on acceptance

- Open access: articles freely available online

- High visibility within the field

- Retaining the copyright to your article

Submit your next manuscript at $\mathbf{s p r i n g e r o p e n . c o m ~}$ 